\title{
Contextos
}

\section{Producción, consumo de información e impacto de la Revista de Arquitectura (Bogotá). Análisis bibliométrico 2016-2019}

\author{
Production, consumption of information and impact in the Revista de Arquitectura (Bogotá). \\ Bibliometric Analysis 2016-2019
}

\author{
César Eligio-Triana \\ Universidad Católica de Colombia. Bogotá (Colombia) \\ Facultad de Diseño, Programa de Arquitectura \\ Centro de investigaciones CIFAR
}

Eligio-Triana C. (2020). Producción, consumo de informació e impacto de la Revista de Arquitectura (Bogotá). Análisis bibliométrico 2016-2019. Revista de Arquitectura (Bogotá), 22(I), 3-17. https://doi.org/10.14718/RevArq.2020.3029

\author{
César Eligio-Triana \\ Arquitecto, Universidad Católica de Colombia. \\ Magíster en Arquitectura, Universidad Nacional de Colombia. \\ Par evaluador e investigador Asociado reconocido por Colciencias (20I5-2020). \\ Representante, Andes Norte, Asociación de Revistas Latinoamericanas de Arqui- \\ tectura (ARLA) \\ Director, Centro de investigaciones CIFAR, Facultad de Diseño, Universidad \\ Católica de Colombia. \\ http://scienti.colciencias.gov.co:808I/cvlac/visualizador/generarCurriculoCv. \\ do?cod rh $=0000$ I55322 \\ https://scholar.google.es/citations?user=CNQtorwAAAAJ\&hl=es \\ (D) http://orcid.org/0000-000I-6620-6392 \\ celigio@ucatolica.edu.co; cesareligioarq@gmail.com
}

\section{Introducción}

\section{Resumen}

La arquitectura tiene un amplio campo de acción disciplinar, que abarca las ciencias sociales, el arte, las humanidades y la ingeniería, por lo cual se busca caracterizar las prácticas de producción editorial, consumo de información e impacto presentes en la Revista de Arquitectura (Bogotá). Para lograrlo, se hizo un estudio de carácter descriptivo y retrospectivo, a partir de indicadores bibliométricos, tomado artículos originales, revisados por pares y publicados en las tres secciones principales de la publicación. Como resultado, se identificó una publicación constante y homogénea en las tres secciones, pero con diferencias en la dinámica de producción y el consumo de información; es en la sección TMS donde hay mayor número de referencias bibliográficas y un mejor índice de Price; también se destaca el uso de revistas y de literatura en inglés. En la sección CUE se observó concentración de la literatura en español, así como un equilibro entre las fechas de las referencias cada cinco años. Para el caso de PAU, se observaron mayor internacionalización y una preferencia por el libro y el uso de referencias de fechas con más de quince años. En general, fueron observables un impacto positivo por citación y métricas alternativas.

Palabras clave: Altmetrics; análisis de revistas; bibliometría; evaluación de revistas; factor de impacto; indicadores bibliométricos; investigación en arquitectura; producción científica

\begin{abstract}
Architecture has a wide range of disciplinary action that encompasses social sciences, art and humanities, and engineering. Thus, this paper aims to characterize the practices of editorial production, information consumption and impact present in the Revista de Arquitectura (Bogotá). To achieve this end, a descriptive and retrospective study was carried out, based on bibliometric indicators and founded on original articles, peer reviewed and published in the three main sections of the journal. As a result of this, a constant and homogeneous publication was identified in the three sections, but with differences in the dynamics of production and consumption of information, being in the TMS section where there is a greater number of bibliographic references and a better Price index; also, the use of journals and literature in English stands out. In the CUE section, a concentration of literature in Spanish was observed, and a balance between the dates of the references every five years. In the case of PAU, a greater internationalization and a preference for books and use of references dated over 15 years was observed. In general, a positive impact was observed by citation and alternative metrics.
\end{abstract}

Keywords: altmetrics; journals analysis; bibliometrics; journal evaluation; impact factor; bibliometric indicators; architecture research; scientific production Evaluado: diciembre $1 / 2019$

La arquitectura como disciplina tiene diferentes formas de comprensión y ámbitos de acción. La clasificación nacional de Colciencias-Publindex (en concordancia con los planteamientos de la Organización para la Cooperación y el Desarrollo Económicos [OCDE]), la incluye dentro de las Humanidades/artes. El SCImago Journal Rank/ Journal Citation Report (SJR/JCR) y Web of Science (WOS) la clasifica como parte de la ingeniería. Adicionalmente, el campo de acción profesional del arquitecto implica un quehacer multidisciplinar e interdisciplinar (Moreno-Toledano, 2015, p. 92) que combina los estudios urbanos (ciencias sociales), el proyecto arquitectónico y urbano (humanidades/artes/diseño) y el componente constructivo y tecnológico (ingeniería). Por ello, se hace necesario, desde estudios bibliométricos, reconocer cómo es la dinámica investigativa de cada uno de estos campos y cómo, a partir del material publicado en la Revista de Arquitectura (Bogotá), se puede llegar a representar las prácticas investigativas disciplinares, $y$, adicionalmente, cómo este material puede aportar a la construcción del corpus disciplinar.

Para lo planteado, se hace importante identificar el comportamiento y la caracterización de cada una de las principales secciones que componen la Revista de Arquitectura (Bogotá): Cultura y espacio urbano (CEU); Proyecto arquitectónico y urbano (PAU), y Tecnología, medioambiente y 
sostenibilidad (TMS); ello, mediante el estudio de la composición, la relevancia, el impacto y la calidad de los artículos publicados. Este tipo de análisis permitirá comparar el comportamiento de Revista de Arquitectura (Bogotá) con revistas regionales (en español) o de corriente principal, con el fin de generar acciones de mejora y orientaciones editoriales que permitan seguir consolidando la Revista de Arquitectura (Bogotá) como un referente en su campo disciplinar.

En este escenario, y al cumplirse 20 años de la primera edición de la Revista de Arquitectura (Bogotá), se justifica hacer un balance de lo que esta publicación ha representado en los procesos de comunicación del conocimiento en la disciplina y cómo ha aportado al urbanismo, la arquitectura y la construcción de edificios.

\section{Las revistas de arquitectura, urbanismo y construcción de edificios en la producción y el consumo de conocimiento}

La producción de conocimiento se construye y se soporta en trabajos previamente desarrollados, que van aportado interpretaciones, métodos y resultados. Para comprender las dinámicas de una disciplina se hace importante, en primer lugar, reconocer los trabajos previos que, a su vez, reconocen los problemas de los cuales se ocupan las comunidades académicas, así como qué interpretaciones y respuestas se han dado. En segundo lugar, se necesita reconocer las dinámicas de producción de conocimiento y cuáles son los aportes y el impacto que generan los nuevos conocimientos publicados. En tercer lugar, reconocer cómo esta nueva producción se vuelve base y soporte para nuevos desarrollos, para así cerrar un ciclo entre la generación, el uso/consumo y la producción de nuevo conocimiento.

De esta manera, "la producción científica no puede avanzar sino apoyándose en los trabajos precedentes, siendo los trabajos actuales la base de futuras investigaciones y publicaciones" (Álvarez, Lorenzo, Andrés, \& Rodríguez, 2007, p. 248). Es así como algunos de los criterios de selección de las referencias están condicionados por la afinidad conceptual y el entorno geográfico.

Por lo anterior, en un artículo de investigación original, el tipo de referencias, la cantidad y el origen ponen de manifiesto cómo los autores buscan evidenciar, justificar y discutir los resultados obtenidos. Y aunque no existe un mínimo o un máximo de referencias, es importante que un autor tenga en cuenta algunas preguntas que puedan orientar la selección y la inclusión del material de referencia y citación: ¿qué comunidad académica o científica está publicando sobre el mismo tema (a favor o en contra)? ¿En qué teorías, conceptos y alternativas estudio y soluciones existentes se basará la interpretación de los resultados? ¿Qué discusión (comparativa, crítica o analítica) se establece con el conocimiento actual en el artículo?

A fin de comprender estas dinámicas, no obstante, al hacer una revisión bibliográfica de los análisis bibliométricos llevados a cabo para revistas de arquitectura, urbanismo o construcción de edificios ${ }^{1}$, los resultados son escasos. A la fecha se identifican algunos trabajos realizados para Informes de la Construcción (Sorli-Rojo \& Mochón-Bezares, 2014), Materiales de construcción (Sorli-Rojo \& Mochón-Bezares, 2013a) y como diagnóstico de las Revistas españolas de arquitectura, ciencias de la construcción y urbanismo: visibilidad e internacionalidad (Sorli Rojo \& Mochón Bezares, 2013b). Más reciente y en el contexto latinoamericano está el artículo Características de la producción científica de la Revista INVI en la era SciELO, 2009-2016 (Medina, Mejías \& Pérez, 2017); también hay estudios ya sobre conceptos particulares, como La gentrificación en los estudios urbanos: una exploración sobre la producción académica de las ciudades (Mendoza, 2016). Por el contrario, en diversas disciplinas se han identificado las características de su producción, y para varias revistas se hicieron estudios bibliométricos, entre los que se destaca el realizado a la Revista Española de Salud Pública, el cual se dividió en tres partes: la primera correspondió a indicadores generales (Pérez Andrés, Estrada Lorenzo, Villar Álvarez, \& Rebollo Rodríguez, 2002). La segunda correspondió a la productividad de los autores y la procedencia institucional y geográfica (Estrada Lorenzo, Villar Álvarez, Pérez Andrés, \& Rebollo Rodríguez, 2003). La tercera analiza las referencias bibliográficas (Álvarez et al., 2007). Estos documentos servirán de insumo para la construcción conceptual, el diseño metodológico y la estructuración e interpretación de resultados.

En tal sentido, cobra importancia la realización de estudios bibliométricos que permitan obtener instrumentos de gestión, seguimiento y evaluación adicionales y alternativos a los ya establecidos, con el fin de tener una radiografía más amplia que permita caracterizar los diferentes campos de conocimiento.

\section{Metodología}

Se hace un estudio de carácter descriptivo y retrospectivo, a partir de indicadores bibliométricos (Ardanuy, 2012; Herrán-Páez, 2019; Peralta González, Maylín, \& Gregorio Chaviano, 2015), tomado los artículos originales, revisados por pares

\footnotetext{
1 Las ecuaciones de búsqueda fueron: Analysis AND Bibliome* AND journals OR architecture OR Urban planning OR construction OR buildings y Bibliometric AND information consumption. Se consultaron los resultados en español disponibles en Scopus, Scielo Redalyc y Google escolar.
} 
y publicados en la Revista de Arquitectura (Bogotá) en las tres secciones principales: Cultura y espacio urbano (CEU); Proyecto arquitectónico y urbano (PAU), y Tecnología, medioambiente y sostenibilidad (TMS). El periodo de estudio es el periodo 2016-2019 (los cuatro últimos años, y el año desde el cual se publica semestralmente la revista). Las fuentes información de artículos, autores, país, filiación y referencias se tomaron de la plataforma de gestión editorial $(\mathrm{OJS})^{2}$, donde están cada uno de los volúmenes, los números y los artículos publicados. Los datos de impacto asociados a citación y métricas alternativas se recuperaron de bases de datos citacionales, como Scopus, Redib y diferentes rankings, y de agregadores como Google Scholar, mediante el uso de Publish or Perish (POP) ${ }^{3}$.

Los indicadores por reconocer fueron:

- Producción: Número de artículos publicados; Procedencia de los artículos publicados (País, Filiación); Coautorías: Cohesión/colaboración y género de los autores.

- Consumo de información: Tomado como referencia la obra de Álvarez et al.: "El análisis del consumo de la información se realiza a partir de las referencias bibliográficas de los trabajos publicados, entendiendo que éstas representan la información científica que han utilizado quienes han realizado una investigación" (2007); por ello, de las referencias bibliográficas de los artículos originales se extrajo la información relativa al año y a la fuente de publicación, al tipo de documento y al idioma, y se calcularon los correspondientes índices de Price (Price, 1963), así como el de obsolescencia. Previendo las dinámicas propias de la disciplina frente al consumo de información, se definió en una ventana más amplia, por lo cual el índice de Price fue complementado con intervalos de cada cinco años. Índice de Price: < 5 años, entre 6 y 10 años, entre 11 y 15 años $y>16$ años.

https://editorial.ucatolica.edu.co/index.php/RevArq

3 https://harzing.com/resources/publish-or-perish
- Impacto: Posicionamiento en los sistemas de indexación y resumen (SIR), niveles de citación abiertos y en revistas de "corriente principal" e Índice H (Grupo Scimago, 2006). En el ámbito nacional, se tomó la información disponible en Publindex y SIIMER; a escala regional se tomaron dos SIR clasificados como bases bibliográficas con comité científico de selección (BBCS) REDIB y REDALYC ${ }^{4}$, y a escala internacional se hizo la identificación de citas en Scopus mediante dos mecanismos: uno, por medio de la opción Advanced Search-REFSRCTITLE, depurando manualmente el resultado, y la segunda, mediante la consulta de Google Scholar (Torres-Salinas, Ruiz-Pérez \& Delgado-López-Cózar, 2009) mediante POP y las alertas de citación de artículos generadas en el perfil de $\mathrm{GS}^{5}$.

Para cada uno de los artículos publicados, la información se tabuló en hojas de cálculo de Excel@ , y el análisis de la información se consolidó por año y sección, a fin de poder determinar diferencias en el comportamiento de cada una de las secciones.

\section{Resultados}

\section{Indicadores de producción}

\section{Artículos publicados entre 2016 y 2019}

Desde 2016 se modificó la periodicidad de la Revista de Arquitectura (Bogotá) a semestral; adicionalmente, se trabajó por mantener el equilibrio entre el número de artículos de las secciones CEU, PAU y TMS; estas fueron el centro de la publicación. Las otras secciones (Contextos/Editorial, Desde la Facultad, y Textos) solo publicaron, como máximo, un artículo por número. De acuerdo con ello, para el periodo 2016-2019 se publicaron en total 81 artículos, y en las 3 secciones principales, 58, como se describe en la figura 1.

4 No se incluyó Scielo, ya que la publicación fue aceptada en agosto de 2018 y a la fecha las métricas para la Revista de Arquitectura (Bogotá) son muy incompletas. http://www.scielo. org.co/scielo.php?script=sci serial\&pid=1657-0308

5 https://scholar.google.es/ citations? user $=$ NhZkGVkAAAAJ $\& h l=e s \& o i=a o$

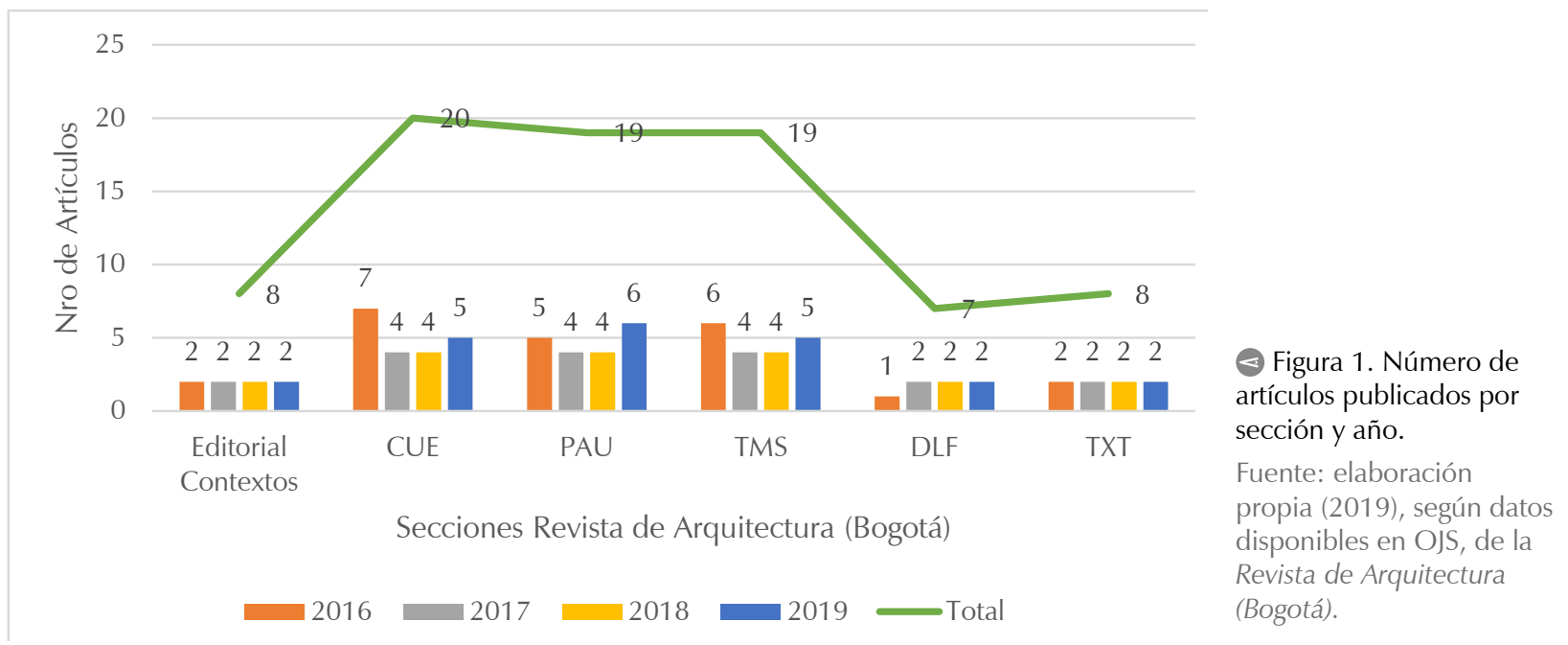




\section{A. Origen total de los artículos}

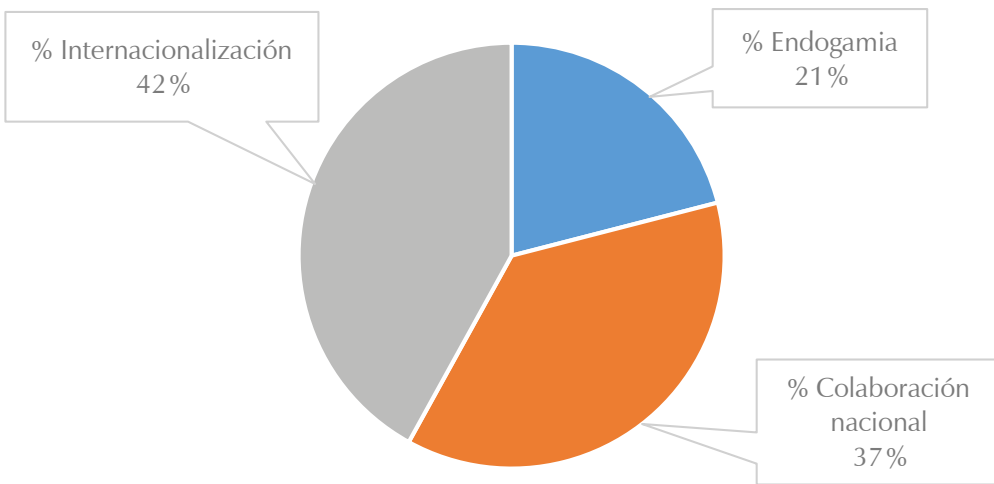

- \% Endogamia

- \% Colaboración nacional

- \% Internacionalización
B. Origen de los artículos en las secciones CEU, PAU y TMS

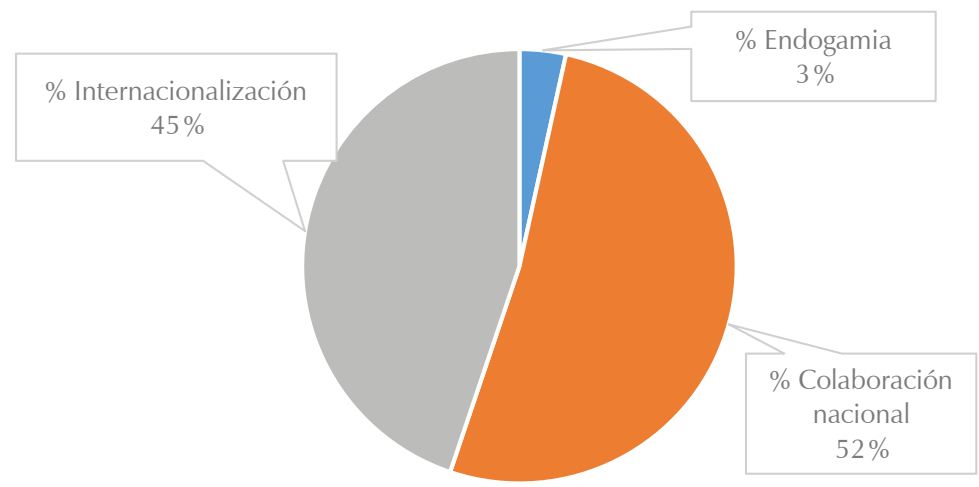

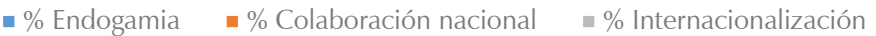

(A) A Figura 2. Origen de los artículos publicados (A) Total, incluyendo todas las secciones (B) solo en las tres secciones principales (CEU, PAU, TMS)

Fuente: elaboración propia (2019).

A

$\rightarrow$ Figura 3 A y B. Origen $\nabla$ (país) de los autores de los artículos publicados.

Fuente: elaboración propia (2019).

B

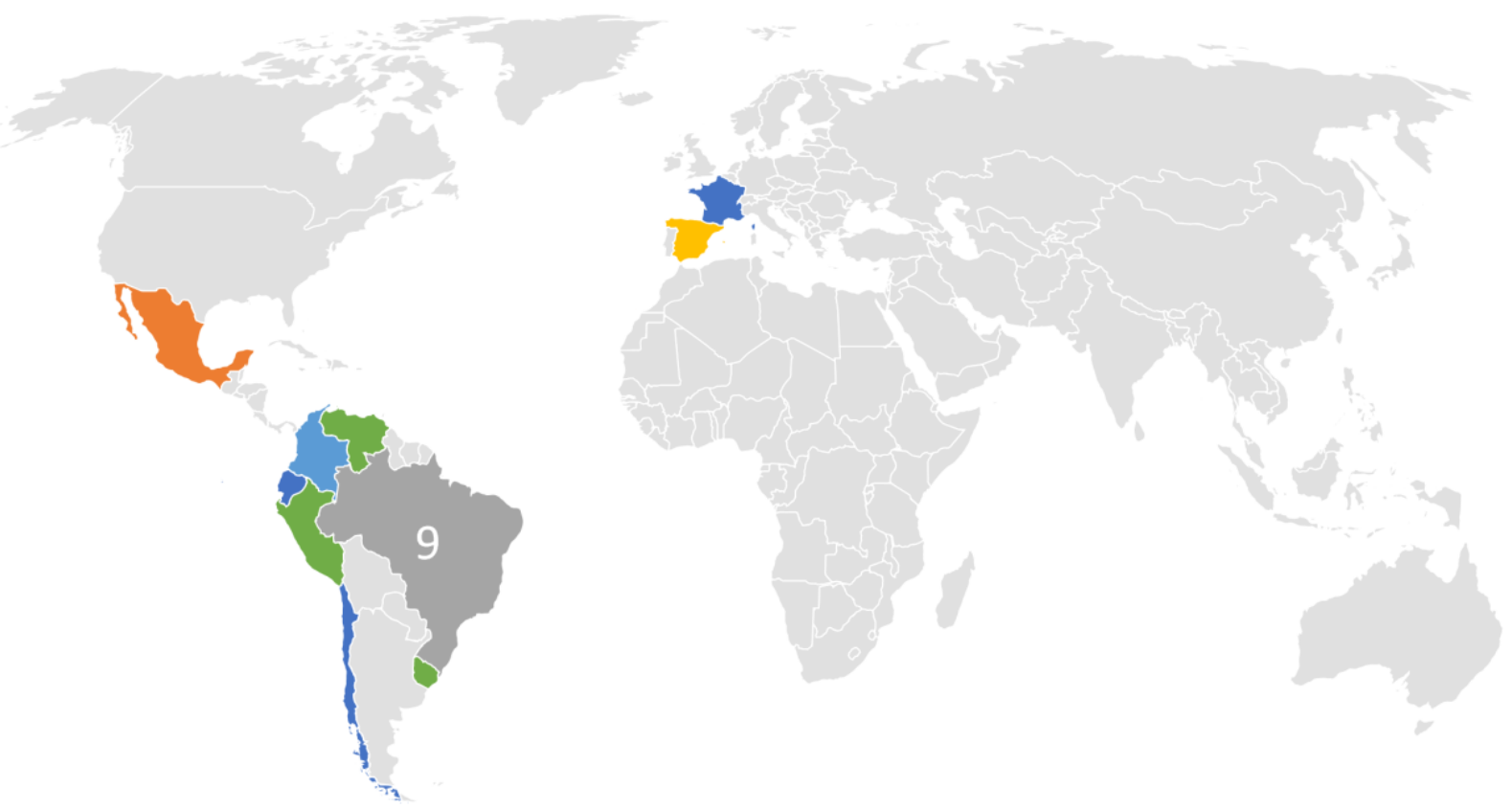

Respecto a la producción publicada en la revista, a esta siempre se la consideró un espacio de discusión que fuera un punto de encuentro de diferentes saberes, por lo cual, se buscó que existiese un equilibrio entre la producción interna, la nacional y la internacional; por tal motivo, y como se observa en la figura 2, de los 58 artículos publicados, 26 (45\%) corresponden a autores internaci onales; 30 (52\%) son de instituciones colombianas y otras 2 (3\%) son internos, debido a las restricciones de endogamia. En cuanto al nivel de internacionalización de cada una de las secciones, PAU lidera con el $58 \%$, seguida de TMS, con el $42 \%$, y, finalmente, CEU con el $35 \%$. 


\begin{tabular}{|c|c|c|c|c|c|c|c|c|c|c|}
\hline \multirow{2}{*}{ Año } & \multirow{2}{*}{ Sección } & \multicolumn{4}{|c|}{ Coautoría de los artículos } & \multicolumn{5}{|c|}{ Número de autores por artículo } \\
\hline & & Sin coautoría & Institucional & Nacional & Internacional & 1 & 2 & 3 & 4 & 5 \\
\hline 2019 & CEU & & 3 & 2 & & & 3 & 2 & & \\
\hline 2018 & CEU & 2 & 1 & & 1 & 2 & & 2 & & \\
\hline 2017 & CEU & 3 & 1 & & & 3 & 1 & & & \\
\hline \multirow[t]{3}{*}{2016} & CEU & 4 & 2 & 1 & & 4 & 1 & 2 & & \\
\hline & Total sección CEU & 9 & 7 & 3 & 1 & 9 & 5 & 6 & $\mathbf{0}$ & 0 \\
\hline & Porcentaje & $45 \%$ & $35 \%$ & $15 \%$ & $5 \%$ & $45 \%$ & $25 \%$ & $30 \%$ & $0 \%$ & $0 \%$ \\
\hline 2019 & PAU & 5 & 1 & & & 5 & 1 & & & \\
\hline 2018 & PAU & 3 & & & 1 & 3 & & 1 & & \\
\hline 2017 & PAU & 2 & 1 & & 1 & 2 & 1 & 1 & & \\
\hline \multirow[t]{3}{*}{2016} & PAU & 3 & 2 & & & 3 & 1 & & 1 & \\
\hline & Total sección PAU & 13 & 4 & $\mathbf{0}$ & 2 & 13 & 3 & 2 & 1 & $\mathbf{0}$ \\
\hline & Porcentaje & $68 \%$ & $21 \%$ & $0 \%$ & $11 \%$ & $68 \%$ & $16 \%$ & $11 \%$ & $5 \%$ & $0 \%$ \\
\hline 2019 & TMS & 1 & 2 & & 2 & 1 & & 2 & 1 & 1 \\
\hline 2018 & TMS & 2 & 2 & & & 2 & 1 & 1 & & \\
\hline 2017 & TMS & & 3 & 1 & & & 2 & 1 & 1 & \\
\hline \multirow[t]{3}{*}{2016} & TMS & 3 & 3 & & & 3 & 2 & 1 & & \\
\hline & Total sección TMS & 6 & 10 & 1 & 2 & 6 & 5 & 5 & 2 & 1 \\
\hline & Porcentaje & $32 \%$ & $53 \%$ & $5 \%$ & $11 \%$ & $32 \%$ & $26 \%$ & $26 \%$ & $11 \%$ & $5 \%$ \\
\hline & $\begin{array}{r}\text { Total Revista de } \\
\text { quitectura (Bogotá) }\end{array}$ & 28 & 21 & 4 & 5 & 28 & 13 & 13 & 3 & 1 \\
\hline & Porcentaje & $48 \%$ & $36 \%$ & $7 \%$ & $9 \%$ & $48 \%$ & $22 \%$ & $22 \%$ & $5 \%$ & $2 \%$ \\
\hline
\end{tabular}

Como se puede observar en la figura $3 \mathrm{~A}$, los autores de los artículos publicados tienen origen en diez países; México y Brasil son con los que mayor colaboración se presenta. En segundo orden de recurrencia se destacan Chile y Ecuador, en América del Sur, y España y Francia, en Europa. Con colaboración esporádica se puede observar a Uruguay, Venezuela y Perú, por lo cual, en la figura $3 \mathrm{~B}$ se diferencia la sección de PAU, que tiene participación de países europeos y la menor participación de autores locales (nacionales).

\section{Tipos de autoría}

En cuanto a los indicadores de colaboración generados por la producción en coautoría, se identificó que el $48 \%$ de los artículos no tienen coautoría, pues corresponden a artículos publicados por un solo autor; la sección de PAU es la que tiene el indicador más alto al respecto, con el $68 \%$. La siguiente tendencia corresponde a la coautoría institucional, con el $36 \%$, que pone de manifiesto la cohesión entre investigadores de una misma institución, y donde TMS es la que más contribu- ciones de este tipo presenta, con el $53 \%$, y PAU, la que menos, con el $21 \%$. El grado de coautoría nacional e internacional es muy similar, con el $7 \%$ y el $9 \%$, respectivamente, lo que pone en evidencia la poca apertura hacia otras instituciones del país de origen o fuera de este (tabla 1).

Con base en lo expuesto, se observa cómo el número de autores que firman el artículo tiene un alto porcentaje en la autoría unipersonal, con el $48 \%$; el $44 \%$, con 2 o 3 autores, y muy pocos casos, con 4 o 5 autores ( $5 \%$ y $2 \%$ ). Estos resultados también marcan una tendencia muy particular en la disciplina, ya que cada día se espera la consolidación de equipos de investigación en los cuales haya cohesión interna y colaboración externa (tabla 1).

En cuanto al género de los autores (tabla 2), se identificó un porcentaje mayoritariamente masculino, con el $64 \%$ versus el $36 \%$ de participación femenina; la sección de PAU fue donde más participación masculina se evidenció, con el $76 \%$, y la $\mathrm{CEU}$, donde hubo mayor autoría femenina.
(A) Tabla 1. Coautoría y número de autores de los artículos publicados en las tres secciones principales.

Fuente: elaboración propia (2019). 
$>$ Tabla 2. Género de los autores y promedio de autores por artículo.

Fuente: elaboración propia (2019).

\begin{tabular}{|c|c|c|c|c|c|}
\hline \multirow{2}{*}{ Año } & \multirow{2}{*}{ Sección } & \multicolumn{2}{|c|}{ Género } & \multirow{2}{*}{$\begin{array}{l}\text { Total de } \\
\text { autores }\end{array}$} & \multirow{2}{*}{$\begin{array}{l}\text { Promedio de } \\
\text { autores por } \\
\text { artículo }\end{array}$} \\
\hline & & Masculino & Femenino & & \\
\hline 2019 & CEU & 8 & 4 & 12 & \\
\hline 2018 & CEU & 7 & 1 & 8 & \\
\hline 2017 & CEU & 3 & 2 & 5 & \\
\hline \multirow[t]{3}{*}{2016} & CEU & 3 & 9 & 12 & \\
\hline & Total sección CEU & 21 & 16 & \multirow{2}{*}{37} & \multirow{2}{*}{1,85} \\
\hline & Porcentaje & $57 \%$ & $43 \%$ & & \\
\hline 2019 & PAU & 7 & 0 & 7 & \\
\hline 2018 & PAU & 4 & 2 & 6 & \\
\hline 2017 & PAU & 5 & 2 & 7 & \\
\hline \multirow[t]{3}{*}{2016} & PAU & 6 & 3 & 9 & \\
\hline & Total sección PAU & 22 & 7 & \multirow{2}{*}{29} & \multirow{2}{*}{1,53} \\
\hline & Porcentaje & $76 \%$ & $24 \%$ & & \\
\hline 2019 & TMS & 7 & 9 & 16 & \\
\hline 2018 & TMS & 5 & 2 & 7 & \\
\hline 2017 & TMS & 8 & 3 & 11 & \\
\hline 2016 & TMS & 7 & 3 & 10 & \\
\hline \multicolumn{2}{|c|}{ Total sección TMS } & 27 & 17 & \multirow{2}{*}{44} & \multirow{2}{*}{2,32} \\
\hline Porcentaje & & $61 \%$ & $39 \%$ & & \\
\hline \multicolumn{2}{|c|}{$\begin{array}{l}\text { Total Revistade Arquitectura } \\
\text { (Bogotá) }\end{array}$} & 70 & 40 & \multirow[t]{2}{*}{110} & \multirow[t]{2}{*}{1,90} \\
\hline Porcentaje & & $64 \%$ & $36 \%$ & & \\
\hline
\end{tabular}

\begin{tabular}{|c|c|c|c|c|c|c|c|c|c|c|c|c|c|}
\hline & $\begin{array}{c}\text { Promedio } \\
2016\end{array}$ & Máximo & Mínimo & \begin{tabular}{|c} 
Promedio \\
2017
\end{tabular} & Máximo & Mínimo & $\begin{array}{c}\text { Promedio } \\
2018\end{array}$ & Máximo & Mínimo & \begin{tabular}{|c} 
Promedio \\
2019
\end{tabular} & Máximo & Mínimo & Total \\
\hline CUE & 39 & 80 & 25 & 21 & 27 & 13 & 26 & 35 & 14 & 30 & 36 & 24 & 29 \\
\hline PAU & 26 & 36 & 20 & 26 & 37 & 15 & 28 & 42 & 19 & 28 & 54 & 15 & 27 \\
\hline $\begin{array}{l}\text { Promedio de } \\
\text { referencias }\end{array}$ & 33 & 65 & 19 & 29 & 39 & 18 & 27 & 38 & 16 & 32 & 54 & 19 & 30 \\
\hline
\end{tabular}

(A) Tabla 3. Máximos, mínimos y promedio de referencias por artículo.

Fuente: elaboración propia (2019).
En cuanto al promedio de autores por artículo, a pesar del alto número de autorías individuales, el promedio es de 1,9, con un promedio superior en TMS (2,32), y el mayor número de autores por artículo, con 5.

\section{Consumo de información}

A partir de los 58 de artículos publicados en las secciones principales (CEU, PAU y TMS), se estableció el consumo de información a partir de las 1773 referencias bibliográficas empleadas en los artículos de la Revista de Arquitectura (Bogotá). En la figura 4 se observa cómo la sección de TMS es la que ostenta el número mayor de referencias, seguida por CEU, y, por último, por PAU.

Si se revisa el número de las referencias dentro de cada sección y por cada año, se puede evidenciar un comportamiento homogéneo, con un promedio de 30 referencias por artículo; la sección TMS tuvo los promedios más altos en 2017, con 41; en 2019, con 37, y en 2016, con 34. Por su parte, CEU tuvo 39 en 2016, y el valor más bajo en 2017, con 21. La sección PAU se mantuvo muy cerca del promedio de la misma sección, con valores entre 26 y 28 (tabla 3). 
En cuanto a los umbrales máximos y mínimos del número de referencias (tabla 3), el máximo se ubicó en 80, durante 2016, en las secciones de CEU, en el artículo "Propuesta de un sistema de indicadores para evaluar la calidad visual del paisaje urbano en asentamientos informales" ${ }^{\prime \prime}$, al igual que en la sección TMS, con el artículo "Resiliencia a inundaciones: nuevo paradigma para el diseño urbano" ${ }^{\prime \prime}$, seguido por 72 en 2019, con "Bucle multidisciplinar para la sustentabilidad urbana". Cabe resaltar que estos dos artículos son de la misma autoría: Luis Fernando Molina-Prieto ${ }^{8}$ et al. En cuanto a los mínimos, se encuentran valores de 11, 13, 15. En el caso del valor más bajo (11), este corresponde a un artículo de TMS que da cuenta de la construcción experimental de un sistema transformable tensado plegable ${ }^{9}$.

Los resultados también tienen que ver con el carácter de algunos de los artículos, por cuanto estos cubren desde revisiones, pasando por resultados de investigación básica y aplicada, hasta desarrollos experimentales, lo cual es consecuente con la tipología de proyectos de carácter científico, tecnológico o de innovación definidos por Colciencias (2017a, pp. 11-15, citando a OCDE, 2002, p. 30).

\section{Índice de Price y obsolescencia}

Posteriormente se hizo el análisis de obsolescencia y del Índice de Price para las referencias empleadas en los artículos, y se encontró que, en general, para la Revista de Arquitectura (Bogotá) el Índice de Price es del 21,3\%; adicionalmente, si se revisa la inmediatez del consumo de información, solo 16 referencias de 1773 - y que equivalen al 0,9\%- fueron referencias del mismo año de publicación ${ }^{10}$. Las referencias del año inme-

6 doi: http://dx.doi.org/10.14718/RevArq.2016.18.1.4

7 doi: http://dx.doi.org/10.14718/RevArq.2016.18.2.8

8 https://scholar.google.es

citations? user $=|\mathrm{KOZgSEAAAA} \& \mathrm{~h}|=\mathrm{es} \& \mathrm{o}=\mathrm{ao}$

9 doi: http://dx.doi.org/10.14718/RevArq.2016.18.1.9

10 Aunque esto también tiene que ver con los tiempos relacionados con el proceso de revisión, edición y publicación, y no solo como responsabilidad de los autores. Por ello, la Revista de Arquitectura (Bogotá) adoptó desde 2018 el sistema de publicación anticipada, y a corto plazo se está evaluando la posibilidad de pasar a flujo continuo. diatamente anterior a la fecha de publicación fueron 83 , equivalentes al $4,7 \%$, y para el segundo año, 78, equivalentes al 4,4\%, con un acumulado de solo el $15,7 \%$ para los 3 primeros años y concentrando el consumo entre el tercer y el sexto años. Por ende, se puede afirmar que estos datos son, en gran medida, un reflejo del comportamiento disciplinar frente a las prácticas investigativas, el acceso y el uso de publicaciones seriadas y la difícil adopción para la disciplina de métricas relacionadas con el factor de impacto.

Por lo anterior, se consideró importante detallar más a fondo cómo es el comportamiento de cada sección. En el caso de CEU, el consumo se puede considerar homogéneo para todos los periodos, oscilando entre el $20,2 \%$ y el $29,7 \%$ y concentrando el $46,6 \%$ en los 10 primeros años (tabla 4).

(7) Figura 4. Número de (7) referencias bibliográficas empleadas en los artículos de las tres secciones principales. Fuente: elaboración propia (2019)

(7) Figura 5. Distribución del (7) número de referencias por año de antigüedad respecto al artículo publicado.

Fuente: elaboración propia (2019)
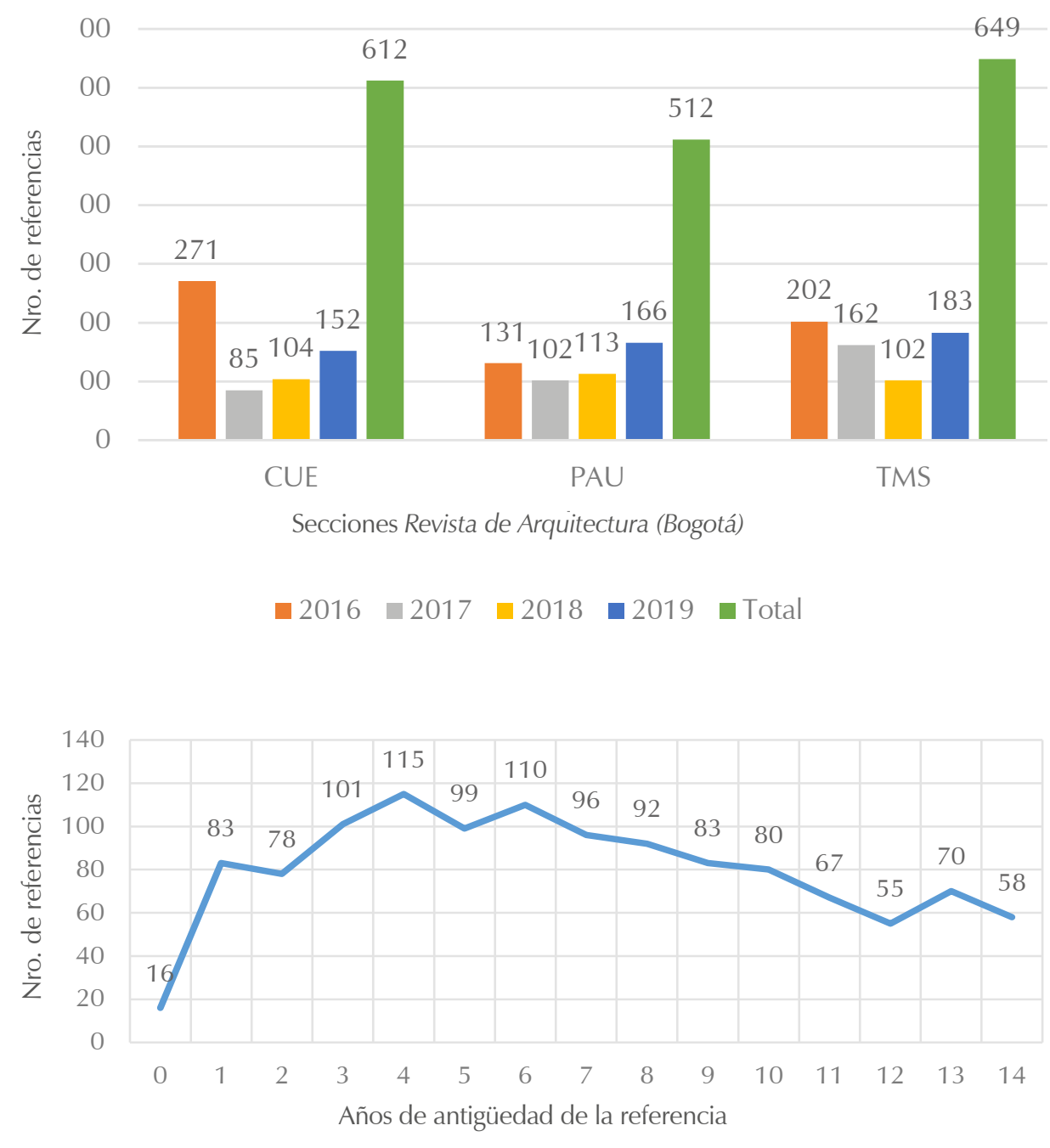

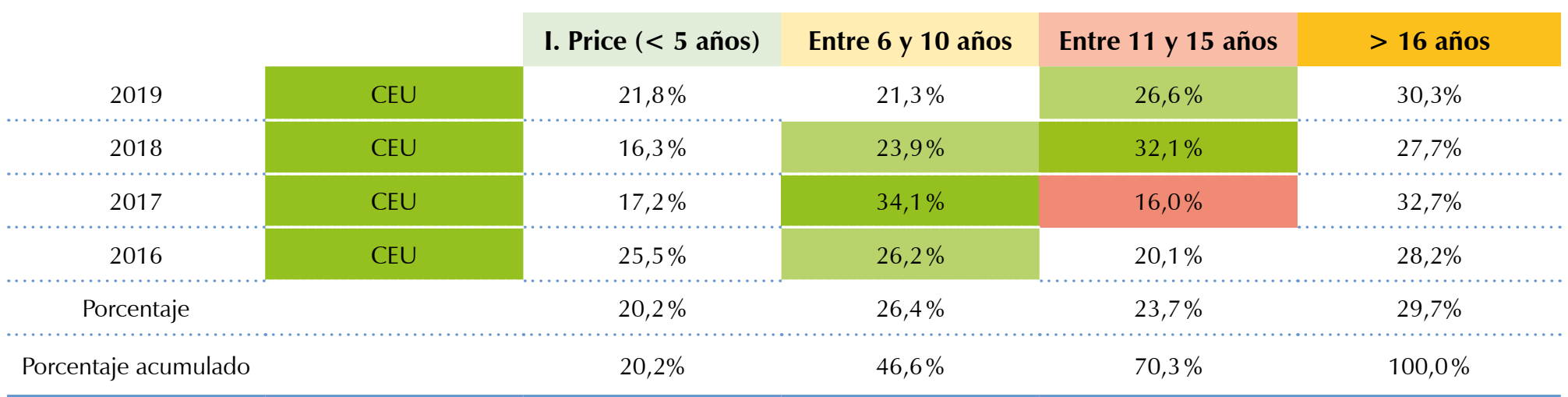




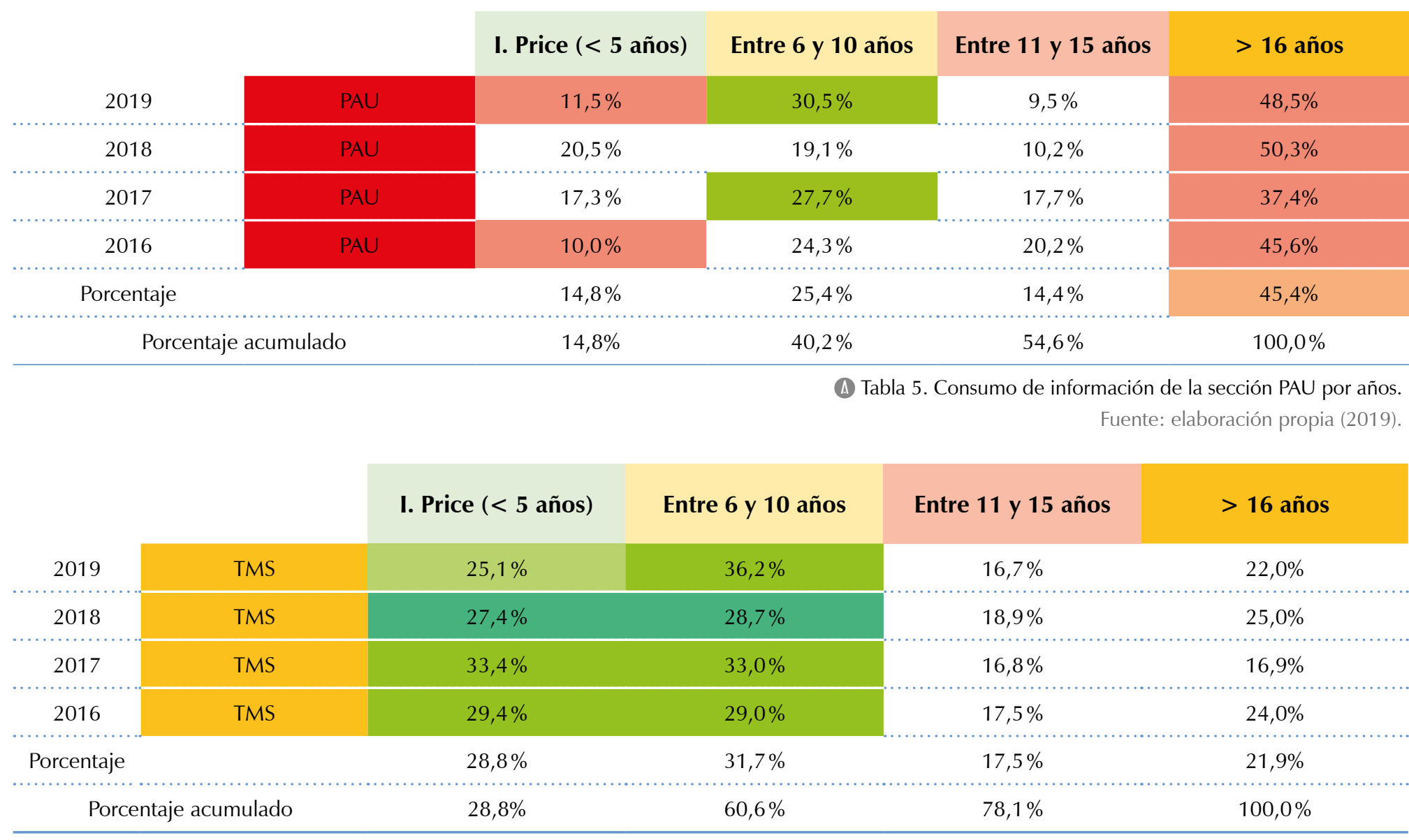

(A) Tabla 6. Consumo de información de la sección TMS por años. Fuente: elaboración propia (2019).

\begin{tabular}{|c|c|c|c|c|c|}
\hline Tipo de documento & $\begin{array}{l}\text { Número de } \\
\text { referencias }\end{array}$ & Porcentaje total & Porcentaje CUE & Porcentaje PAU & Porcentaje TMS \\
\hline Libro & 749 & $43,3 \%$ & $42,2 \%$ & $50,6 \%$ & $37,2 \%$ \\
\hline Revista & 528 & $28,8 \%$ & $28,8 \%$ & $17,4 \%$ & $40,2 \%$ \\
\hline Cap. libro & 97 & $6,1 \%$ & $5,9 \%$ & $9,5 \%$ & $2,8 \%$ \\
\hline Documento institucional & 83 & $4,5 \%$ & $6,3 \%$ & $2,4 \%$ & $4,8 \%$ \\
\hline Página web & 76 & $3,7 \%$ & $3,4 \%$ & $4,2 \%$ & $3,6 \%$ \\
\hline Normas/doc. legal/ley & 67 & $3,6 \%$ & $6,6 \%$ & $1,1 \%$ & $3,3 \%$ \\
\hline Página web institucional & 47 & $2,3 \%$ & $2,1 \%$ & $1,8 \%$ & $2,9 \%$ \\
\hline Tesis maestría & 26 & $1,4 \%$ & $1,1 \%$ & $1,6 \%$ & $1,7 \%$ \\
\hline Figura/ilustración & 22 & $1,6 \%$ & $1,2 \%$ & $1,8 \%$ & $2,0 \%$ \\
\hline Tesis doctorado & 19 & $1,1 \%$ & $0,3 \%$ & $2,3 \%$ & $0,6 \%$ \\
\hline Memorias, congreso evento & 16 & $0,8 \%$ & $0,8 \%$ & $1,5 \%$ & $0,0 \%$ \\
\hline Fotografía & 16 & $1,1 \%$ & $0,3 \%$ & $2,7 \%$ & $0,1 \%$ \\
\hline Archivo & 10 & $0,7 \%$ & $0,0 \%$ & $2,0 \%$ & $0,0 \%$ \\
\hline Software & 7 & $0,4 \%$ & $0,3 \%$ & $0,2 \%$ & $0,7 \%$ \\
\hline Tesis pregrado & 4 & $0,3 \%$ & $0,3 \%$ & $0,4 \%$ & $0,2 \%$ \\
\hline Entrevista & 3 & $0,1 \%$ & $0,2 \%$ & $0,2 \%$ & $0,0 \%$ \\
\hline Otro & 2 & $0,1 \%$ & $0,1 \%$ & $0,2 \%$ & $0,0 \%$ \\
\hline Proyecto arq./urbano & 1 & $0,1 \%$ & $0,2 \%$ & $0,0 \%$ & $0,0 \%$ \\
\hline
\end{tabular}

(A) Tabla 7. Tipos de documento identificados en las listas de referencias. Fuente: elaboración propia (2019). 
Para el caso de la sección PAU, esta tiene los valores más bajos para el Índice de Price, con solo el 14,8\%; además, Ilama la atención que el $45,4 \%$ de las referencias tienen más de 16 años (tabla 5).

En la sección TMS, se observa el Índice de Price más alto $(28,8 \%$ ), y que concentra el $60,6 \%$ de las referencias entre los 10 primeros años (tabla 6).

\section{Tipos de documentos presentes en las} referencias

En la distribución de las referencias por tipo de documento, como se muestra en la tabla 7 , se identificaron diversas tipologías y su grado de recurrencia. El libro resultó ser la fuente más consultada, con el $43,3 \%$; y si a este se suman los capítulos de libro, el promedio llega al 49,4\%. En segundo lugar se encuentran las revistas, con el 28,8\% ${ }^{11}$. Los documentos instituciones disponibles en sitios web o como normas representan el $10,4 \%$. Las tesis de pregrado y de posgrado, por su parte, corresponden al 2,8\%.

De acuerdo con las dinámicas actuales de circulación del conocimiento, que privilegian a la revista por sobre el libro, también se hizo un análisis dentro de cada sección, el cual evidenció una tendencia ascendente de CUE en el uso de revistas, el cual pasó del $28 \%$ en 2016 al 42,8\% en 2019. En el caso de PAU, el promedio de uso de revistas durante el periodo objeto de estudio fue del $17,4 \%$, con un máximo del $22,9 \%$ en 2016 y un mínimo del $13,3 \%$ en el 2019; dichos valores estuvieron muy por debajo del promedio de la Revista de Arquitectura (Bogotá) (28,8\%), lo cual refleja un uso bajo de revistas y una tendencia a la baja. La sección TMS, a diferencia de las otras 2 secciones, mantuvo un promedio del $40,2 \%$, el cual fue relativamente estable durante el periodo en mención y obtuvo un máximo del 45,7\% en 2017.

11 Cabe aclarar que en dicho valor no se distinguió entre revista de investigación (indexadas o no) y revistas de divulgación.
En cuanto al idioma de las referencias consultadas, se confirmó que el español es el idioma dominante, con el 55,9\% seguido por el $31,8 \%$ en inglés, y el portugués, con el 8,6\%. En este punto es importante resaltar que la sección TMS tiene un comportamiento diferente, pues el $54,8 \%$ de las referencias son en inglés, lo que ratifica una dinámica diferente de la de las otras 2 secciones; tal vez, por la transversalidad entre lo local, lo regional y lo internacional de los temas abordados, lo cual también podría explicar por qué CEU es la que usa mayoritariamente referencias en español $(75,2 \%)$, ya que, por lo general, su objeto de estudio aborda temas principalmente de las ciudades en el contexto latinoamericano (tabla 8).

\section{Impacto}

Una vez identificados el perfil de la producción y el consumo de información de la Revista de Arquitectura (Bogotá), se analizó cómo fueron recibidos los contenidos publicados por las comunidades académicas. Para esto, lo primero fue analizar el número y el tipo de sistemas de indexación y resumen (SIR) ${ }^{12}$ (tabla 9) en los cuales se halla presente la publicación, ya que aportan a la divulgación y a la visibilidad, y hacen que la revista sea reconocida por su calidad editorial y científica atendiendo los estándares actuales de publicación del conocimiento científico.

En el listado de la tabla 9 y en el ámbito local, se destaca la indexación en Publindex en Categoría $\mathrm{B}$, ya que, como resultado de la convocatoria 768 de 2016 y del cambio de modelo que ahora valora el impacto (medido en citación), salieron el $79 \%$ de las revistas de arquitectura y urbanismo (Eligio-Triana, 2018, p. 7). Frente a los resultados de la Convocatoria 830 de $2018^{13}$, se mantiene la

12 Según la clasificación emitida por Colciencias (2017b) en el Documento de actualización de los sistemas de indexación y resumen-SIR

13 https://minciencias.gov.co/convocatorias/investigacion/convocatoria-para-indexacion-revistas-cientificas-colombianas-0
Tabla 8. Idioma de las referencias.

Fuente: elaboración propia (2019)

\begin{tabular}{|c|c|c|c|c|c|c|c|}
\hline Sección & Español & Inglés & Portugués & Francés & Italiano & Otro & $\begin{array}{c}\text { No. de } \\
\text { referencias }\end{array}$ \\
\hline \multirow{2}{*}{ CEU } & 469 & 100 & 40 & 2 & 0 & 1 & \multirow{2}{*}{612} \\
\hline & $75,2 \%$ & $17,7 \%$ & $6,7 \%$ & $0,3 \%$ & $0,0 \%$ & $0,2 \%$ & \\
\hline \multirow{2}{*}{ PAU } & 290 & 109 & 59 & 45 & 7 & 2 & \multirow{2}{*}{512} \\
\hline & $55,1 \%$ & $22,9 \%$ & $13,2 \%$ & $7,1 \%$ & $1,3 \%$ & $0,3 \%$ & \\
\hline \multirow{2}{*}{ TMS } & 238 & 354 & 43 & 13 & 0 & 1 & \multirow{2}{*}{649} \\
\hline & $37,5 \%$ & $54,8 \%$ & $5,9 \%$ & $1,6 \%$ & $0,0 \%$ & $0,1 \%$ & \\
\hline \multirow{2}{*}{$\begin{array}{c}\text { Total Revista de } \\
\text { Arquitectura } \\
\text { (Bogotá) }\end{array}$} & 997 & 563 & 142 & 60 & 7 & 4 & \multirow{2}{*}{1773} \\
\hline & $55,9 \%$ & $31,8 \%$ & $8,6 \%$ & $3,0 \%$ & $0,4 \%$ & $0,2 \%$ & \\
\hline
\end{tabular}




\begin{tabular}{|c|c|c|c|c|c|c|c|c|c|c|c|}
\hline Año & 2008 & 2009 & 2010 & 2011 & 2012 & 2013 & 2014 & 2015 & 2016 & 2017 & 2018-2019 \\
\hline \multicolumn{12}{|c|}{ Nacional } \\
\hline & $\begin{array}{l}\text { Publindex } \\
\text { Cat C }\end{array}$ & $\begin{array}{l}\text { Publindex } \\
\text { Cat C }\end{array}$ & $\begin{array}{l}\text { Publindex } \\
\text { Cat C }\end{array}$ & $\begin{array}{l}\text { Publindex } \\
\text { Cat C }\end{array}$ & $\begin{array}{l}\text { Publindex } \\
\text { Cat C }\end{array}$ & $\begin{array}{l}\text { Publindex } \\
\text { Cat C }\end{array}$ & $\begin{array}{l}\text { Publindex } \\
\text { Cat B }\end{array}$ & $\begin{array}{l}\text { Publindex } \\
\text { Cat B }\end{array}$ & $\begin{array}{l}\text { Publindex } \\
\text { Cat B }\end{array}$ & $\begin{array}{l}\text { Publindex } \\
\text { Cat B }\end{array}$ & $\begin{array}{l}\text { Publindex } \\
\text { Cat B }\end{array}$ \\
\hline \multicolumn{12}{|c|}{ Regional } \\
\hline BBCS & & & & & & & & & & & SciELO \\
\hline BBCS & & & & & & & & & Redib & Redib & Redib \\
\hline BBCS & & & Redalyc & Redalyc & Redalyc & Redalyc & Redalyc & Redalyc & Redalyc & Redalyc & Redalyc \\
\hline BBCS & & & & Clase & Clase & Clase & Clase & Clase & Clase & Clase & Clase \\
\hline BBCS & & Dialnet & Dialnet & Dialnet & Dialnet & Dialnet & Dialnet & Dialnet & Dialnet & Dialnet & Dialnet \\
\hline $\mathrm{N} / \mathrm{A}$ & Latindex-C & Latindex-C & Latindex-C & Latindex-C & Latindex-C & Latindex-C & Latindex-C & Latindex-C & Latindex-C & Latindex-C & Latindex-C \\
\hline $\mathrm{N} / \mathrm{A}$ & & ARLA & ARLA & ARLA & ARLA & ARLA & ARLA & ARLA & ARLA & ARLA & ARLA \\
\hline $\mathrm{N} / \mathrm{A}$ & & & & & & & & & & LatinREV & LatinREV \\
\hline \multicolumn{12}{|c|}{ Internacional } \\
\hline IB & & & & & & & & & & ECSI & ECSI \\
\hline BBCS & & & & & & & & & & DOAJ & DOAJ \\
\hline BBCS & & & & & EBSCO & EBSCO & EBSCO & EBSCO & EBSCO & EBSCO & EBSCO \\
\hline$N / A$ & & & & & & & & MIAR & MIAR & MIAR & MIAR \\
\hline
\end{tabular}

(A) Tabla 9. Histórico de clasificación en Sistemas de Indexación y Resumen (SIR) de la Revista de Arquitectura (Bogotá), y tendencia ascendente de inclusión. Fuente: Plataforma Publindex y cada uno de los SIR..

$\rightarrow$ Figura 6. Resultados del ranking bibliométrico de revistas colombianas.

Fuente: SIIMER (2019).

$\rightarrow$ Figura 7. Resultados generales de la Revista de Arquitectura (Bogotá) en el ranking de revistas Redib. Fuente: Redib (2018).

Ranking bibliométrico de revistas colombianas, según SIIMER

\begin{tabular}{|c|c|c|c|c|c|c|c|c|c|c|c|c|}
\hline \multicolumn{5}{|c|}{ Institución } & \multicolumn{3}{|c|}{ Ärea } & \multicolumn{2}{|l|}{ Nivel } & & & \\
\hline \multicolumn{3}{|c|}{ Seleccione una opción } & & $\cdot$ & \multicolumn{2}{|c|}{ Humanidades } & v & Seleccione un_. & & Filtrar & Limpiar & \\
\hline Mostrar & $50 \quad$ regist & & & & & & & \multicolumn{2}{|c|}{ Buscar: } & & & \\
\hline \multicolumn{2}{|r|}{ Institución } & لII & \multicolumn{2}{|l|}{ Revista } & & $\begin{array}{l}\text { Indice } \\
\text { de Osk }\end{array}$ & Nivel $\downarrow$ & \multicolumn{2}{|c|}{$\begin{array}{c}\text { Tiempo promedio } \\
\text { de aceptación } \\
\text { (dias) }\end{array}$} & \multicolumn{3}{|c|}{ Item Preferencial $\downarrow$} \\
\hline \multicolumn{3}{|c|}{ Universidad Católica de Colombia } & Revista de arquitectura & & 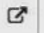 & 168.63 & & 01 & $\mathrm{n} / \mathrm{d}$ & & & $n / d$ \\
\hline
\end{tabular}

Ranking de Revistas REDIB

\begin{tabular}{|c|c|c|c|}
\hline Edición & Clasificación & $\begin{array}{l}\text { Cobertura } \\
\text { completa }\end{array}$ & $\begin{array}{l}\text { Percentil del Factor } \\
\text { de Impacto Norma- } \\
\text { lizado }\end{array}$ \\
\hline
\end{tabular}

$2018 \quad 78$

$2017 \quad 63$

posición en categoría $B$, y se destaca el retorno al índice Bibliográfico Nacional de la revista Apuntes.

En el ámbito internacional, en la consulta de MIAR $^{14}$ y al filtrar por campo de conocimiento ARQUITECTURA, a esta se la clasifica entre el grupo de mayor puntaje $(9<11)$, con una valoración de 9,8 a escala internacional, y está en primer lugar entre las revistas colombianas incluidas. En el caso

$14 \mathrm{El}$ sistema crea una matriz de correspondencia entre las revistas, identificadas por su ISSN, y las bases de datos y repertorios que las indizan o las incluyen.

\begin{tabular}{|c|c|c|c|}
\hline \multicolumn{4}{|c|}{ Powerwd by $\begin{array}{c}\text { Clarivate } \\
\text { Analytics }\end{array}$} \\
\hline $\begin{array}{l}\text { Porcentaje ajusta- } \\
\text { do de citas en la } \\
\text { materia }\end{array}$ & $\begin{array}{l}\text { Procentaje de } \\
\text { artículos más } \\
\text { citados }\end{array}$ & $\begin{array}{l}\text { Percentil } \\
\text { medio }\end{array}$ & $\begin{array}{l}\text { Calificador } \\
\text { global }\end{array}$ \\
\hline 3.343 & 6.315 & 7.932 & 24.48 \\
\hline 4.959 & 6.667 & 6.278 & 24.439 \\
\hline
\end{tabular}

del Emerging Sources Citation Index (ESCI) ${ }^{15}$, solo están presentes 44 revistas (2017) en el campo de la arquitectura, cifra que aumentó a 46 en 2018, y donde solo participan 2 colombianas.

En segundo lugar, se reconocieron diversos rankings y métricas donde la Revista de Arquitectura (Bogotá) participa, con el fin de determinar el impacto de la publicación frente a revistas del

\section{Ver:http://mjl.clarivate.com/cgi-bin/jrnlst/jliresults.} $\mathrm{cgi} ? \mathrm{PC}=\mathrm{EX} \& \mathrm{SC}=\mathrm{BK}$ 


\section{Revista}

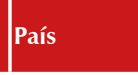

Percentil del Factor de

Porcentaje de artículos

Porcentaje ajustado de
citas en la materia

Porcentaje de artícu-
los mas citados

Percentil

Calificador

Revista de Arquitectura Colombia

93.38728324

8.421052632

3.343811408

6.15789414

7.93248936224 .48008522

92 ACE: architecture. city and environment

España

92.91907514

8.823529412

5.350098253

VLC arquitectura. Research Journal*

España

97.15799615

6

1.003143422

125 EGA. Revista de expresión gráfica

España

91.1849711

5.262100084

8.693909661

3.529411765

7.98328235323 .72107939

España

88.48747592

2.941176471

1.337524563

$3.361344538 \quad 4.81109887422 .66068498$

$5.79552 \quad 23.19133191$

$2.941176471 \quad 2.72602205919 .6866751$

(B)
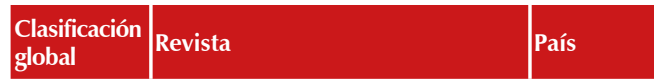

Percentil del Factor

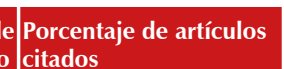

Colombia

96.38728324

263 Dearq

274 Arquiteturarevista

371 Territorios

459 Revista de Direito da Cidade
Colombia

Brasil

Colombia

Brasil
86.84971098

80.97302505

60.26011561

52.74566474
8.421052632

1.657458564

3.448275862

10.92436975

7.575757576

\section{Porcentaje ajustado de
citas en la materia}

3.343811408

1.003143422

1.337524563

0.392931983

0.936991652

\begin{tabular}{c|l|l|}
\begin{tabular}{|c|l|} 
Porcentaje de artícu- \\
los mas citados
\end{tabular} & $\begin{array}{l}\text { Percentil } \\
\text { medio }\end{array}$ & $\begin{array}{l}\text { Calificador } \\
\text { Global }\end{array}$ \\
\hline 6.315789474 & 7.932489362 & 24.48008522 \\
1.104972376 & 1.532906077 & 18.42963828 \\
2.298850575 & 3.189195402 & 18.24937429 \\
0 & 5.489435593 & 15.41331059 \\
0 & 4.184142424 & 13.08851128
\end{tabular}

0

4.18414242413 .08851128
(C)
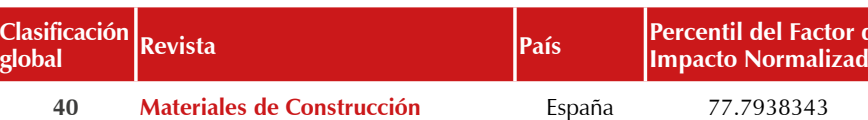
Impacto Normalizad

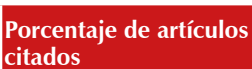

España

77.7938343

78 Revista de Arquitectura

274 Arquiteturarevista

299 Informes de Construcción

Colombia

Brasil

96.38728324

80.97302505

España

60.45279383

3.448275862

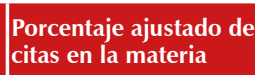

0.786712881

8.421052632

18.31325301
3.343811408

1.337524563

0.898853219

Porcentaje de artícu-
los mas citados
0

6.315789474

2.298850575

0.240963855
Percentil

Calificador

18.2858833326 .78995277

$7.932489362 \quad 24.48008522$

3.18919540218 .24937429

7.41096144617 .46336507 mismo campo de conocimiento y áreas similares; para esto se tomó como referencia el grado de citación.

La primera fuente de consulta corresponde a Google Scholar $(G S)^{16}$, en el cual la revista se consultó por 2 vías. La primera en el listado de publicaciones TOP en español, donde, por su índice de citación, en 2018 y 2019 fue incluida en las posiciones 77 y 81 , respectivamente, junto con revistas de todos los campos de conocimiento. Para 2019, el valor del Índice h5 fue de 17, y la Mediana h5, de 34. En segundo lugar, según la consulta por $\mathrm{POP}^{17}$, el h5 pasó de 7 en 2016 a 18 en 2018, y en 2019 el resultado fue de 14 . Como ya se indicó, los resultados de GS requieren un proceso de lectura e interpretación más detallado. Debido a ello, se pueden observar diferentes tendencias: por una parte, se observó la citación de preprint (tesis de pregrado y de posgrado y documentos institucionales) a artículo y consolidados a escala de repositorios institucionales de carácter interno, nacional e internacional; por otro, se evidenciaron citas de artículo a artículo y consolidados a escala de revista, sin diferenciar el nivel de indexación de estas.

16 Frente a la exactitud y la presión de GS, se afirma que no hace ningún filtro de la información e incluye todos los documentos accesibles en la web y que están disponibles en dominios académicos; por lo tanto, se afirma que el índice $\mathrm{H}$ de GS no es selectivo ni exhaustivo, pero de igual manera mide las publicaciones en igualdad de condiciones, pero se hace necesario un proceso más detallado de lectura e interpretación de los resultados.

17 Según los cálculos realizados para 2016 y 2018 por Colciencias/Publindex, usando el software Publish or Perish. https://harzing.com/resourcespublish-or-perish?source $=$ pop_ 7.15 .2643 .7260
En segundo lugar, se revisaron los resultados obtenidos en GS frente a otros rankings y SIR. En el ámbito nacional, se consultó Sistema Integral de Medición de Revistas Científicas (SIIMER) ${ }^{18}$, en el cual se destacan los siguientes aspectos de la metodología al hacer ciertas precisiones, que ayudan a interpretar de mejor manera los resultados de GS. Las revistas con valor $\geq 20 \%$ en autocitaciones en relación con el total de las citas obtenidas fueron excluidas, en aras de reducir el sesgo de los resultados del índice. En la fórmula de cálculo final, las citas internas y las autocitas tienen una ponderación baja frente a las citas externas $(x 4)$; como indicador de calidad y visibilidad y en síntesis el Índice de Osk se calculó de la siguiente manera:

$$
\begin{aligned}
& \text { Índice de Osk }=\left(\operatorname{Inv}^{*} 1+\mathrm{Ai}+\mathrm{An}+\mathrm{Ae}\right)+\left(\operatorname{Rev}^{*}\right. \\
& 0,5+\mathrm{Ai}+\mathrm{An}+\mathrm{Ae})+\left(\operatorname{Ref}^{*} 0,25+\mathrm{A}+\mathrm{An}+\mathrm{Ae}\right)+ \\
& \text { ediciones+(ciex*4)+(Ac*1) })^{19}
\end{aligned}
$$

Como resultado de este cálculo, la Revista de Arquitectura (Bogotá) ocupó para 2018 el primer lugar en el área de humanidades (figura 7).

En el ámbito regional, los resultados obtenidos en Redib y Redalyc son los siguientes: en el caso de Redib, se consideran relevantes los datos, ya que

18 Es un producto financiado por la Universidad del Magdalena, a través del programa Fonciencias (2017), de la Vicerrectoría de Investigación. El ranking se basó en la medición de las revistas indexadas en el Índice Bibliográfico Nacional Publindex-Convocatoria 768 de 2016, y a la fecha no hay resultados de la Convocatoria 830 de 2018. https://siimer.com/

19 Donde: el número total de artículos de investigación (Inv); de revisión (Rev); de reflexión (Ref); reportes de casos (Rec); ediciones (Ed); citas externas (Ciex); citas internas o autocitas (Ac) y los autores, centrándose especialmente en su afiliación; es decir, autores locales o internos (Ai), autores nacionales (An) y autores extranjeros o internacionales (Ae).
(A) Figura 8. Resultados de la Revista de Arquitectura (Bogotá) en las materias de arquitectura (A), urbanismo $(B)$ y construcción y tecnología de los edificios (C). Fuente: Redib (2018). 
$\theta$ Figura 9. Citas recibidas en Scopus.

Fuente: elaboración propia (2019), con datos de GS y de Scopus.

$\rightarrow$ Figura 10. Distribución de citas por fechas, recibidas en Scopus.

Fuente: elaboración propia (2019), con datos de GS y de Scopus.
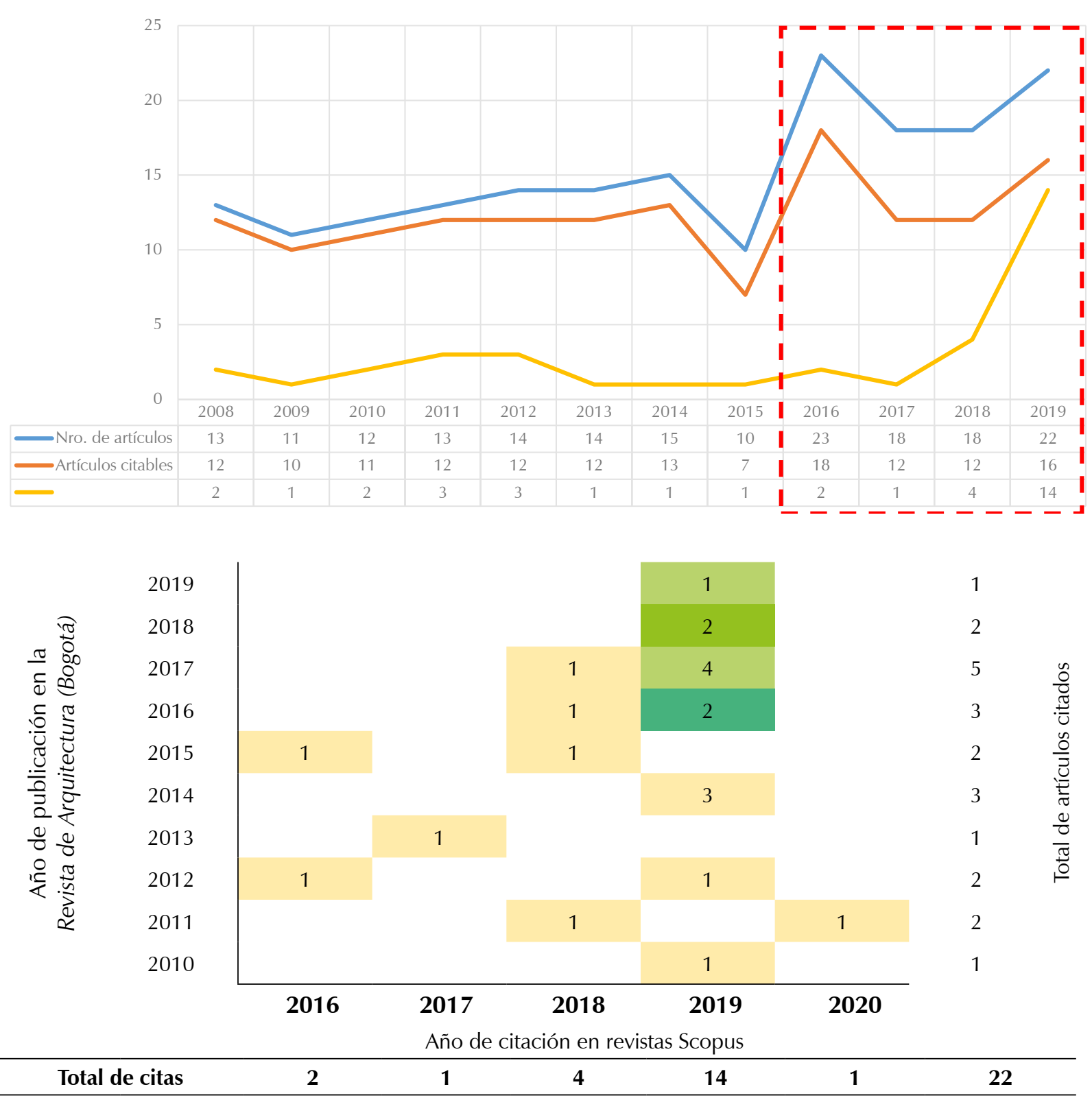

\begin{tabular}{|c|c|c|c|c|c|c|c|c|c|}
\hline Revista & País & $\begin{array}{l}\text { Artículos } \\
\text { externos }\end{array}$ & $\begin{array}{c}\text { Artículos en } \\
\text { coautoría } \\
\text { externa }\end{array}$ & $\begin{array}{l}\text { Internacio- } \\
\text { nalización }\end{array}$ & $\begin{array}{l}\text { Índice de } \\
\text { esfuerzo } \\
\text { editorial }\end{array}$ & $\begin{array}{l}\text { Semanas } \\
\text { recepción - } \\
\text { aceptación }\end{array}$ & Descargas & $\begin{array}{l}\text { Países que } \\
\text { descargan }\end{array}$ & $\begin{array}{l}\text { Decil de } \\
\text { descarga }\end{array}$ \\
\hline $\begin{array}{l}\text { Revista de Arquitectura } \\
\text { (Bogotá) }\end{array}$ & Colombia & $37 \%$ & $33,1 \%$ & G3 & 0,54 & 33,42 & $417 \mathrm{k}$ & 63 & 4 \\
\hline Arquiteturarevista & Brasil & $53,5 \%$ & $39 \%$ & G2 & 0,56 & 38,68 & $175 \mathrm{k}$ & 77 & 7 \\
\hline Dearq & Colombia & $51,2 \%$ & $28,1 \%$ & G2 & 0,72 & 25,54 & $156 k$ & 58 & 7 \\
\hline Oculum Ensaios & Brasil & $10,1 \%$ & $43,8 \%$ & G3 & 0,63 & 28,56 & $41 \mathrm{k}$ & 42 & 9 \\
\hline Arquitectura y Urbanismo & Cuba & $21,9 \%$ & $38,2 \%$ & G3 & 1,21 & 20,31 & $200 \mathrm{k}$ & 59 & 6 \\
\hline $\begin{array}{l}\text { Revista Legado de } \\
\text { Arquitectura y Diseño }\end{array}$ & México & $8,2 \%$ & $62,7 \%$ & G4 & 0,39 & 13,73 & $135 \mathrm{k}$ & 51 & 7 \\
\hline
\end{tabular}

${ }^{1}$ https://www.redalyc.org/revista.oa?id=1251\&tipo= coleccion

(A) Tabla 10. Comportamiento de las revistas de arquitectura presentes en Redalyc.

Fuente: Redalyc (2019).
[...] se incluyen aquellas revistas indexadas simultáneamente en REDIB y en los diferentes índices de la Web of Science Core Collection: Science Cltation Index (SCI), Social Sciences Citation Index (SSCl), Arts \& Humanities Cltation Index (A\&HCI) y Emerging Sources Citation Index (ESCI). ${ }^{20}$

20 Powered b y Clarivate Analytics https://redib.org/recursos/ Ranking/Revistas?lng=es
Los datos se basan en las citas de artículos indexados a artículos indexados, agregando las citas artículo-artículo a escala de revista. El resultado final se calcula por el Percentil del Factor de Impacto Normalizado ( $\mathrm{NCl}$ Percentile) y normalizadas como porcentaje dentro de la materia o las materias en que estén incluidas; en tal sentido, el resultado para la Revista de Arquitectura (Bogotá) 
fue: el global ocupó la posición 63 (2017) y la 78 (2018), junto con revistas de todas las áreas (figura 8), y en el ámbito disciplinar ocupó la primera posición (2017 y 2018) en las materias de arquitectura, al igual que en urbanismo, y la segunda posición en construcción y tecnología de los edificios (figura 9).

En el caso de Redalyc, con la nueva versión del modelo (AmeliCa, 2019) y del portal, la implementación de XML y la revaluación de revistas, a futuro se espera tener indicadores alternativos y más detallados. En este nuevo escenario, se resalta que de la colección de Artes y Humanidades/Arquitectura solo hacen parte seis publicaciones, y, por el momento, uno de los datos más representativos, para la Revista de Arquitectura (Bogotá) son las descargas recibidas durante el periodo 2013-2018 del acervo 2005-2017 (tabla 10); y aunque no están relacionadas con los índices de citación ya expuestos, sí reflejan el posible vínculo entre descargas y citación y la implementación de métricas alternativas (Altmetrics), que permiten hacer una valoración más integral de las publicaciones.

El resultado de estos indicadores ubica a la publicación en el primer lugar, con 417k descargas, lo cual representa más del doble de la revisita siguiente, Arquitectura y Urbanismo, con $200 k$, y casi el triple $(2,7)$ frente a la otra revista colombiana presente: DeArq, con 156k. El otro indicador para tener en cuenta corresponde al índice de esfuerzo editorial ${ }^{21}$, con un resultado de 0,54 , el cual se puede considerar bajo; pero, si se lo mira frente a los otros resultados, se podría afirmar que existe un mayor impacto, pues con menos artículos, pero con mayor selectividad, se han logrado mayor descarga y mayor citación. A partir de estos resultados también se considera importante incrementar el número de artículos, la participación externa (internacional) y los niveles de coautoría.

Finalmente, se hizo un análisis de las citas provenientes de revistas de "corriente principal"

21 El índice expresa el promedio de trabajos que genera la revista, tomando como base el promedio de la disciplina a que se adscribe. Así, un índice de 1 indica que genera el mismo promedio de artículos que las revistas de su disciplina; 0,5 indicaría que genera la mitad. tomando como base la información de Scopus, lo cual puso en evidencia una tendencia ascendente para 2019, a la par con el número de artículos publicados a lo largo de los últimos años (figura 9).

Los resultados arrojan un incremento del número de citas para 2019.

Al revisar cómo se distribuyen estas citas, se observó que la inmediatez con la cual se están citando los contenidos publicados en la revista se va reduciendo, pues para 2019 ya se había citado un artículo del mismo año de publicación y con el mayor número de citas para el material publicado en 2017, lo que puede ayudar a mejorar el factor de impacto. Por otra parte, se observa cómo siguen vigentes los contenidos publicados de 2010 a 2013, que mantienen una citación constante (figura 10).

Al desglosar estos datos, también se puede identificar el tipo de revistas que están citando a la Revista de Arquitectura (Bogotá) y, de esta manera, reconocer cómo están siendo aceptados los contenidos entre las comunidades académicas. Si bien es cierto que la mayoría de citaciones provienen de revistas Q4, lo cual es atribuible al idioma español, también se observa una concentración entre el Q3 y el Q2, y en los últimos años, unas citaciones en revista del núcleo principal Q1.

\section{Discusión}

Como se evidenció en la revisión inicial y como afirman Medina et al. (2017, p. 51), estos estudios son de gran valor y relevancia, ya que "no se conoce un análisis pormenorizado de indicadores de actividad de revistas especializadas" en este campo, pero, "al mismo tiempo, hace que dicho valor sea relativo por cuanto se carece de referencias para establecer puntos de comparación y criterios de evaluación pertinentes", por lo cual es difícil hacer un diagnóstico certero de los indicadores expuestos; mucho más, Ilegar a generalizaciones sobre el comportamiento disciplinar. En tal sentido, el estudio bibliométrico hecho a la Revista de Arquitectura (Bogotá) se aproxima a reconocer y caracterizar algunos patrones asociados a la producción del conocimiento científico publicado en la revista y

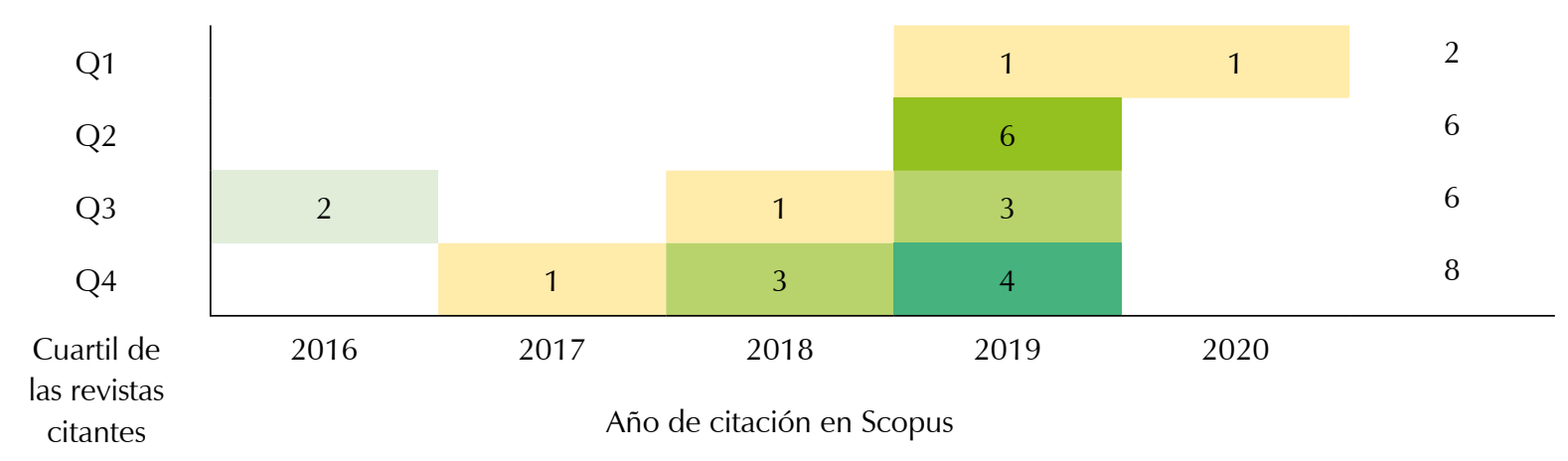

Figura 11. Distribución de citas por año y por cuartil de las revistas citantes.

Fuente: elaboración propia (2019), con datos de GS y de Scopus. 
$\rightarrow$ Figura 12. Co-author index para arquitectura.

Fuente: Grupo de Evaluación de la Ciencia y la Comunicación Científica (EC3), (2015).

\section{Arquitectura}

Año Tipo de revista Moda Media Mediana Máximo № de artículos

\begin{tabular}{lllllll}
2015 & Nacional & 1 & 1.7 & 1 & 12 & 77 \\
\cline { 2 - 6 } & Internacional & 1 & 2.4 & 2 & 8 & 59
\end{tabular}

Distribución de número de autores por artículo - Burbujas Donut $\bigcirc$ Columnas

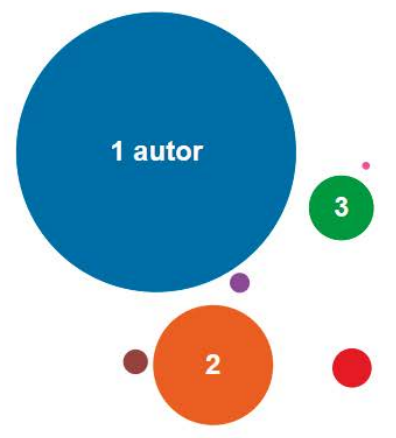

en cada una de sus secciones, a partir de lo cual se pueden establecer algunas particularidades.

La producción consignada en la Revista de Arquitectura (Bogotá) refleja una concentración en la autoría unipersonal, con el $48 \%$, y un promedio de 1,9, lo cual se corresponde con el estudio realizado por el Grupo de Evaluación de la Ciencia y la Comunicación Científica (EC3) en 2015, donde la moda corresponde a 1 y la media oscila entre 1,7 y 2,4 (figura 12). Esto también se corresponde con lo detectado en el estudio de la revista $I N V I^{22}$ (Medina et al., 2017, p. 44), que arrojó el mismo valor (48\%) de autoría individual y un promedio de 1,83 .

En cuanto a la colaboración, siguen existiendo una participación y una publicación local (nacional) marcadas, pero con una tendencia a ir reduciendo la brecha hacia porcentajes más equilibrados con participación regional; sin embargo, también se hace necesario migrar hacia participaciones más internacionales. Esto se debería logar manteniendo el abordaje de los problemas locales.

En relación con el género de los autores, si se comparan los resultados frente al estudio de Medina et al., en el que el " $60 \%$ de los autores es de género masculino (142 autores), mientras que tan solo un $40 \%$ de las autoras es mujer" (2017, p. 46), el comportamiento de la Revista de Arquitectura (Bogotá) es ligeramente similar, con el $64 \%$ de hombres y el $36 \%$ de mujeres; si se lo compara con la Revista Española de Salud Pública, el resultado varía un poco, pero con la misma tendencia: el 56,5\%, hombres, y el 43,5\%, mujeres (Estrada Lorenzo et al., 2003, p. 339). En relación con el consumo de información, los resultados muestran

22 También se considera oportuno hacer comparaciones con la revista INVI, por cuanto es de las pocas publicaciones que tienen estudios bibliométricos, y, adicionalmente, para 2017, en el SIR era Q1 en arquitectura y Q2 en estudios urbanos.
Media de autores por artículo en el periodo 1999-2015

- Media Moda Máximo

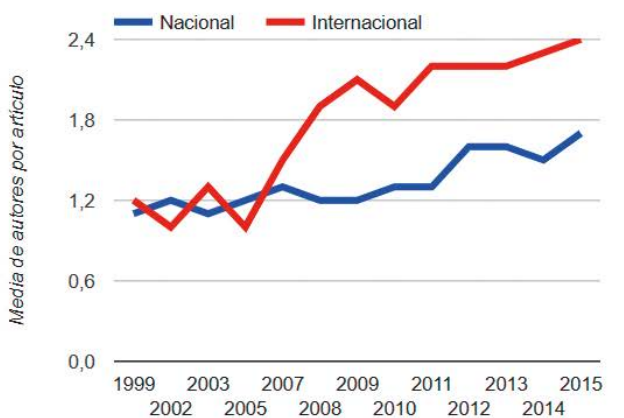

Año

que en las tres secciones se usa un número de referencias que puede considerarse similar; pero se encontraron grandes diferencias al momento de caracterizar cómo están compuestas, y en particular, en lo relacionado con el Índice de Price, ya que este permitió reconocer de mejor manera las diferencias que hay entre los procesos de investigación asociados a cada una de las secciones. La sección TMS se adscribe mejor a la ingeniería, y dicho campo se asocia al componente tecnológico y ambiental, que, a su vez, tiene una gran producción académica, por lo cual se usan más referencias de actualidad publicadas en revistas y en inglés. En el otro extremo, la sección PAU se asocia más al arte y las humanidades, lo cual guarda más relación con el proceso creativo; en este sentido, se observó que hay una tendencia a usar textos clásicos y de más de 16 años, con fuente principal en el libro y en español. En el medio se encuentra la sección $\mathrm{CEU}$, ligada más a las ciencias sociales, y en cuyo caso, por lo general, se observó una relativa homogeneidad en los indicadores estudiados.

En cuanto al número de referencias empleadas por artículo, el valor de la Revista de Arquitectura (Bogotá) fue de 30; dicho valor es superior al promedio de las revistas en español presentes en Scopus, que es de 25, y donde se observa una singularidad en la revista $A R Q$ (Santiago-Chile), la cual solo tiene un promedio de 4,88 Ref./Doc, que corresponde a una práctica muy singular en un sistema que tiene como base la citación.

En cuanto al impacto de la publicación, en las citaciones obtenidas se destaca que los contenidos están siendo usados en muchos documentos disponibles en el ámbito de repositorios, gracias a lo cual asume una contribución a los procesos de investigación formativa y de formación en investigación (Parra, 2004); adicionalmente, se hace notorio el impacto entre las comunidades académicas e investigativas al figurar entre los primeros 
puestos en los rankings regionales. Por otra parte, con el estudio del comportamiento entre revistas de corriente principal se pone en evidencia que la Revista de Arquitectura (Bogotá) está en igualdad de condiciones con revistas presentes en Scopus, y en algunos casos, por encima de ellas.

\section{Conclusiones}

La Revista de Arquitectura (Bogotá) es reconocida como una de las pioneras y líderes en los procesos de indexación en publicaciones en el campo de arquitectura y urbanismo en el ámbito nacional.

Los resultados del impacto, la citación y el Índice $\mathrm{H}$ son producto de una trayectoria consolidada de 20 años, a lo largo de la cual la publicación es reconocida por su calidad editorial y científica en su campo disciplinar y ante los distintos públicos objetivo, lo cual se evidencia al aplicar diferen- tes indicadores cualitativos y cuantitativos y métricas alternativas, frente a los cuales la revista lidera constantemente como muestra del reconocimiento y de su posicionamiento en los medios investigativo y académico.

Dentro del marco de las publicaciones de arquitectura y urbanismo, se hace necesario seguir adelantado estudios más detallados de cada publicación, a escala nacional e internacional, con el fin de seguir caracterizando las prácticas investigativas y de producción de conocimiento, a fin de lograr un equilibrio entre las dinámicas internacionales y las realidades del contexto local en el que consume la información publicada en las revistas de la región. Dichos estudios deben valorar aspectos más allá de lo cuantitativo y valorar el significado de los resultados, para así lograr mejores orientaciones en los procesos de investigación, $y$, de esta manera, fortalecer los procesos de publicación que aporten al nuevo conocimiento.

\section{Referencias}

Álvarez, F. V., Lorenzo, J. M. E., Andrés, C. P., \& Rodríguez, M. J. R. (2007). Estudio bibliométrico de los artículos originales de la revista Española de Salud Pública (19912000). Parte tercera: Análisis de las referencias bibliográficas. Revista Española de Salud Pública, 81(3), 247-259.

https://doi.org/10.1590/S113557272007000300002

AmeliCa. (2019). Métricas Responsables: Reflexión y análisis en torno a la comunicación de la ciencia. Recuperado de:

http://amelica.org/index.php/metricasresponsables/

Ardanuy, J. (2012). Breve introducción a la bibliometría.

https://doi.org/10.1038/nmat3485

Colciencias. (2017a). Tipología de proyectos calificados como de carácter científico (N. $\left.{ }^{\circ} 5\right)$. Recuperado de::

http://www.colciencias.gov.co/sites/default/ files/upload/convocatoria/documento-siresabril-2017.pdf

Colciencias. (2017b). Documento de actualización de los sistemas de indexación y resumen-SIR. Recuperado de::

http://www.colciencias.gov.co/sites/default/ files/upload/convocatoria/documento-siresabril-2017.pdf

Eligio-Triana, C. (2018). El rol de las revistas de arquitectura y urbanismo en la divulgación del conocimiento. Participación e impacto de los SIR. Revista de Arquitectura, 20(1), 3-13. https://doi.org/10.14718/RevArq.2018.20.1.2005

Estrada Lorenzo, J. M., Villar Álvarez, F., Pérez Andrés, C., \& Rebollo Rodríguez, M. J. (2003). Estudio bibliométrico de los artículos originales de la Revista Española de Salud Pública (1991-2000). Parte segunda: Productividad de los autores y procedencia institucional y geográfica. Revista Española de Salud Pública, 77(3), 333-346. https://doi.org/10.1590/s113557272003000300004
Grupo de Evaluación de la Ciencia y la Comunicación Científica (EC3). (2015). Co-author index. Recuperado de::

http://www.coauthorindex.info/layout. php? subject = Arquitectura \& chart_ type $=$ bubbles\&stat $=$ mean

Grupo Scimago. (2006). El índice h de Hirsch: aportaciones a un debate. El profesional de la información, 15(4), 304-306. Recuperado de: http://www.elprofesionaldelainformacion. com/contenidos/2006/julio/8.pdf

Herrán-Páez, E. (2019). Análisis bibliométrico de la producción científica colombiana (2003-2015) https://doi.org/10.3145/colombia scimago

Medina, L. C., Mejías, C. O., \& Pérez, G. C. (2017). Características de la producción científica de la Revista INVI en la era SciELO, 2009-2016. Biblios, 67(67), 42-55. https://doi.org/10.5195/biblios.2017.348

Mendoza, F. R. (2016). La gentrificación en los estudios urbanos: una exploración sobre la producción académica de las ciudades. Cadernos Metrópole, 18(37), 697-719. https://doi.org/10.1590/2236-9996.20163704

Moreno-Toledano, L. A. (2015). Complejidad, transdisciplinariedad y proyecto: Alcances y estrategias para el diseño en el siglo XXI (Universidad de las Américas Puebla). Recuperado de:

http://catarina.udlap.mx/u dl a/tales/ documentos/dct/moreno_t_la/

OCDE. (2002). Manual de Frascati. Medición de las actividades científicas y tecnológicas. Propuesta de norma práctica para encuestas de investigación y desarrollo experimental. Paris: Fundación Española para la Ciencia y la Tecnología (FECYT).

Parra, C. (2004). Apuntes sobre la investigación formativa. Educación y Educadores, 7, 57-77. Recuperado de::

http://educacionyeducadores.unisabana. edu.co/index.php/eye/article/view/54
Peralta González, M. J., Maylín, G. F., \& Gregorio Chaviano, O. (2015). Criterios, clasificaciones y tendencias de los indicadores bibliométricos en la evaluación de la ciencia. Revista Cubana de Información en Ciencias de la Salud, 26(3), 290-309. Recuperado de http://scielo.sld.cu/pdf/ics/v26n3/rci09315.pdf

Pérez Andrés, C., Estrada Lorenzo, J. M., Villar Álvarez, F., \& Rebollo Rodríguez, M. J. (2002). Estudio bibliométrico de los artículos originales de la Revista Española de Salud Pública (1991-2000). Parte primera: Indicadores generales. Revista Española de Salud Pública, 76(6), 659-672.

https://doi.org/10.1590/S1135-57272002000600003

Price, D. J. de S. (1963). Little science, big science. New York: Columbia University Press.

Sorli-Rojo, A., \& Mochón-Bezares, G. (2013a). La revista "Materiales de Construcción", 2003-2012: Un análisis bibliométrico. Materiales de Construccion, 63(312), 613-621. https://doi.org/10.3989/mc.2013.07513

Sorli-Rojo, Á., \& Mochón-Bezares, G. (2014). Informes de la Construcción: un análisis bibliométrico (2007-2013). Informes de la Construcción, 66(536), e041. https://doi.org/10.3989/ic.14.108

Sorli Rojo, Á., \& Mochón Bezares, G. (2013b). Revistas españolas de arquitectura, ciencias de la construcción y urbanismo: visibilidad e internacionalidad. BiD: textos universitaris de biblioteconomia i documentació https://doi.org/10.1344/BiD2013.30.19

Torres-Salinas, D., Ruiz-Pérez, R., \& Delgado-López-Cózar, E. (2009). Google Scholar como herramienta para la evaluación científica. Profesional de la Información, 18(5), 501-510.

https://doi.org/10.3145/epi.2009.sep.03 


\section{Vol.}
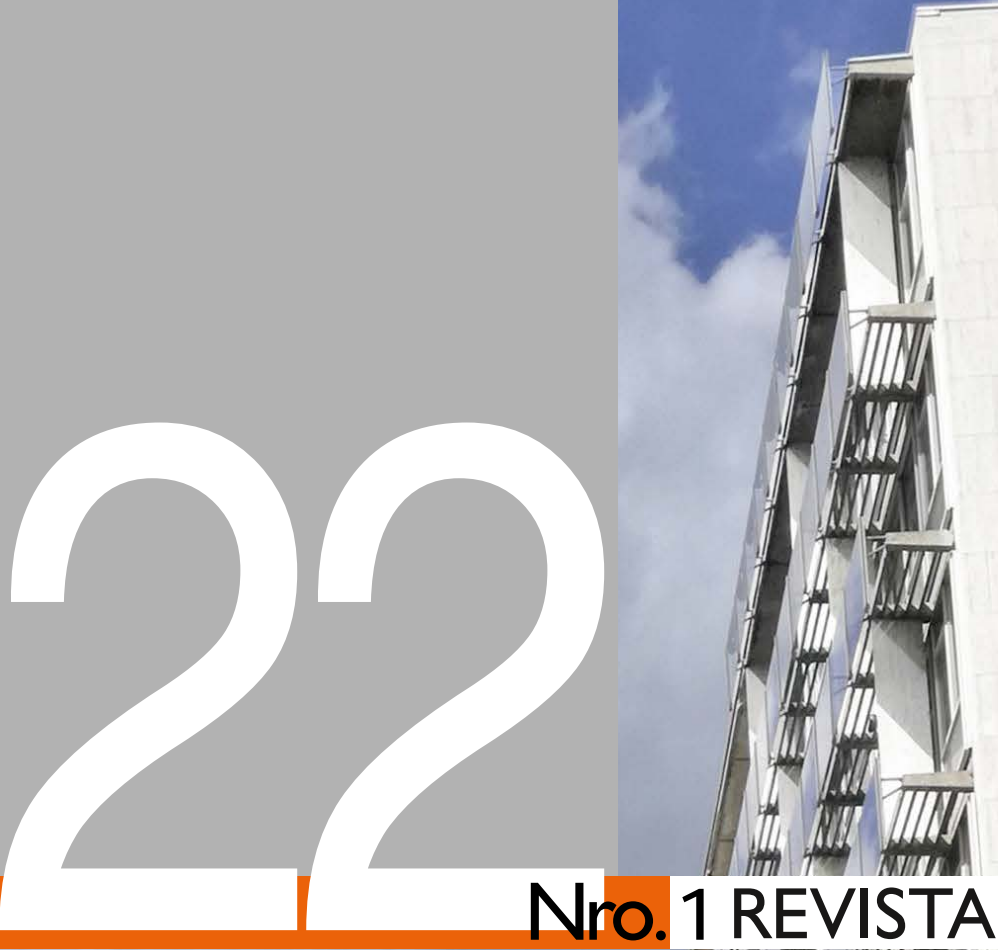

ISSN: 1657-0308 (Impresa)

Nro. 1 REVISTA DE ARQUITECTURA

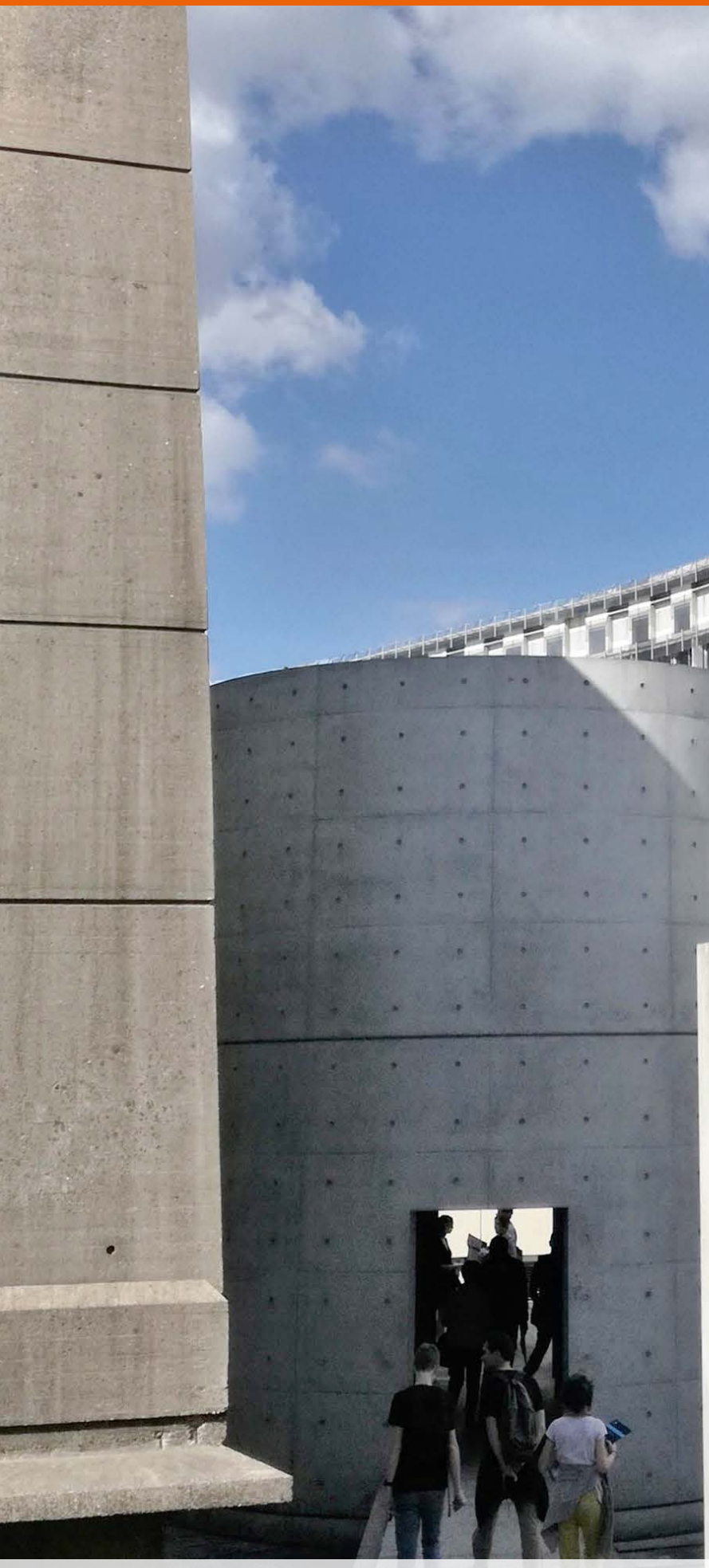

$\overline{9}$
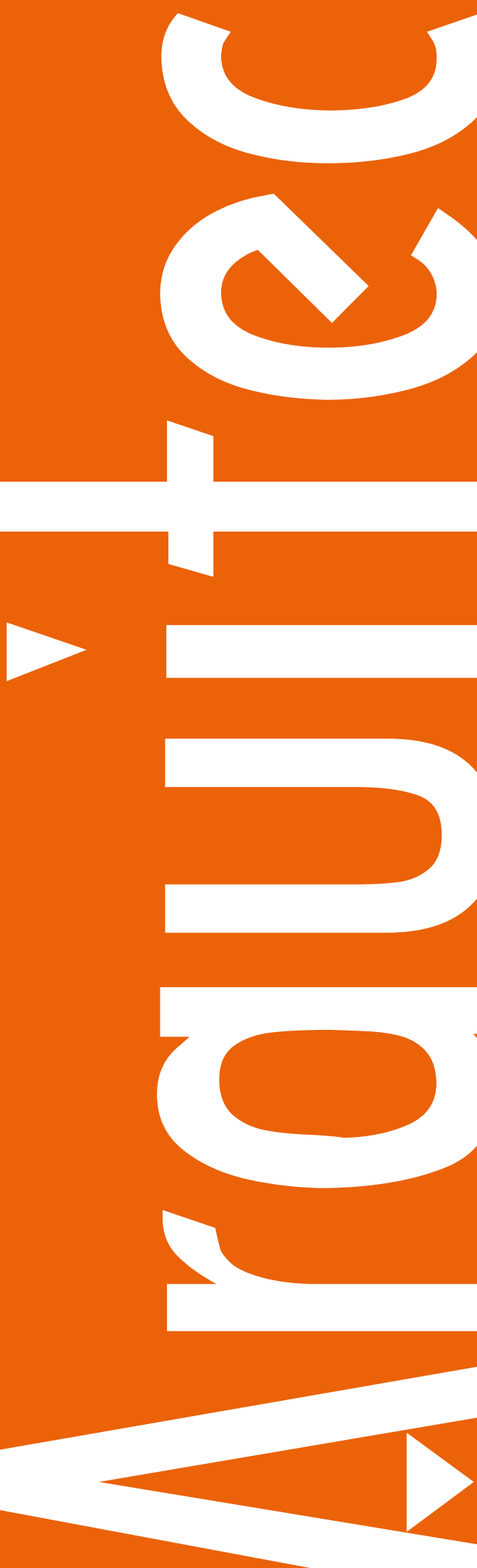


\section{Enfoque y alcance}

La Revista de Arquitectura (Bogotá) ( (ISSN 1657-0308 Impresa y E-ISSN 2357-626X en línea) es una publicación científica seriada de acceso abierto, arbitrada mediante revisión por pares (doble ciego) e indexada, en donde se publican resultados de investigación originales e inéditos.

Está dirigida a la comunidad académica y profesional de las áreas afines a la disciplina. Es editada por la Facultad de Diseño y el Centro de Investigaciones (CIFAR) de la Universidad Católica de Colombia en Bogotá (Colombia).

La principal área científica a la que se adscribe la Revisto de Arquitectura (Bogotá) según la OCDE es:

Gran área: 6. Humanidades

Área: 6.D. Arte

Disciplina: 6D07. Arquitectura y Urbanismo

También se publican artículos de las disciplinas como 2A02, Ingeniería arquitectónica; 5C03, Estudios urbanos (planificación y desarrollo); 6D07, Diseño.

Los objetivos de la Revista de Arquitectura (Bogotá) son:

- Promover la divulgación y difusión del conocimiento generado a nivel local, nacional e internacional

- Conformar un espacio para la construcción de comunidades académicas y la discusión en torno a las secciones definidas.

- Fomentar la diversidad institucional y geográfica de los autores que participan en la publicación.

- Potenciar la discusión de experiencias e intercambios científicos entre investigadores y profesionales.

- Contribuir a la visión integral de la arquitectura, por medio de la concurrencia y articulación de las secciones mediante la publicación de artículos de calidad.

- Publicar artículos originales e inéditos que han pasado por revisión de pares, para asegurar que se cumplen las normas éticas, de calidad, validez científica, editorial e investigativa.

- Fomentar la divulgación de las investigaciones y actividades desarrolladas en la Universidad Católica de Colombia.
Palabras clave de la Revista de Arquitectura (Bogotá): arquitectura, diseño, educación arquitectónica, proyecto y construcción, urbanismo.

Idiomas de publicación: español, inglés, portugués y francés. Título abreviado: Rev. Arquit.

\section{Titulo corto: RevArq}

\section{Políticas de sección}

La revista se estructura en tres secciones correspondientes a las líneas de investigación activas y aprobadas por la institución, y dos complementarias, que presentan dinámicas propias de la Facultad de Diseño y las publicaciones relacionadas con la disciplina.

Cultura y espacio urbano. En esta sección se publican los artículos que se refieren a fenómenos sociales en relación con el espacio urbano, atendiendo aspectos de la historia, el patrimonio cultural y físico, y la estructura formal de las ciudades y el territorio.

Proyecto arquitectónico y urbano. En esta sección se presentan artículos sobre el concepto de proyecto, entendido como elemento que define y orienta las condiciones proyectuales que devienen en los hechos arquitectónicos o urbanos, y la forma como estos se convierten en un proceso de investigación y nuevo de conocimiento. También se presentan proyectos que sean resultados de investigación, los cuales se validan por medio de la ejecución y transformación en obra construida del proceso investigativo. También se contempla la publicación de investigaciones relacionadas con la pedagogía y didáctica de la arquitectura, el urbanismo y el diseño.

Tecnología, medioambiente y sostenibilidad. En esta sección se presentan artículos acerca de sistemas estructurales, materiales y procesos constructivos, medioambiente y gestión, relacionados con los entornos social-cultural, ecológico y económico.

Desde la Facultad. En esta sección se publican artículos generados en la Facultad de Diseño, relacionados con las actividades de docencia, extensión, formación en investigación o internacionalización, las cuales son reflejo de la dinámica y de las actividades realizadas por docentes, estudiantes y egresados; esta sección no puede superar el $20 \%$ del contenido.

Textos. En esta sección se publican reseñas, traducciones y memorias de eventos relacionados con las publicaciones en Arquitectura y Urbanismo.
A Frecuencia de publicación

Desde 1999 y hasta el 2015, la Revista de Arquitectura (Bogotá) publicó un volumen al año, a partir del 2016 se publicarán dos números por año en periodo anticipado, enero-junio y julio-diciembre, pero también maneja la publicación anticipada en línea de los artículos aceptados (versión Post-print del autor).

La Revista de Arquitectura (Bogotá) se divulga mediante versiones digitales (PDF, HTML, EPUB, XML) e impresascon un tiraje de 700 ejemplares, los tiempos de producción de estas versiones dependerán de los cronogramas establecidos por la editorial.

Los tiempos de recepción-revisión-aceptación pueden tardar entre seis y doce meses dependiendo del flujo editorial de cada sección y del proceso de revisión y edición adelantado.

Con el usuario y contraseña asignados, los autores pueden ingresar a la plataforma de gestión editorial y verificar el estado de revisión, edición o publicación del artículo.

\section{A Canje}

La Revista de Arquitectura (Bogotá) está interesada en establecer canje con publicaciones académicas, profesionales o científicas del área de Arquitectura y Urbanismo, como medio de reconocimiento y discusión de la producción científica en el campo de acción de la publicación.

\section{Mecanismo}

Para establecer canje por favor descargar, diligenciar y enviar el formato: RevArq FP20 Canjes
Universidad Católica de Colombia (2020, enero-junio). Revista de Arquitectura (Bogotá), 22(I) I-188. Doi: 10.14718

ISSN: 1657-0308 E-ISSN: 2357-626X

Especificaciones:

Formato: $34 \times 24 \mathrm{~cm}$

Papel: Mate $115 \mathrm{~g}$

Tintas: Negro y policromía
A Contacto

Dirección postal:

Avenida Caracas No. 46-72.

Universidad Católica de Colombia

Bogotá D.C.(Colombia)

Código postal: 111311

Facultad de Diseño Centro de Investigaciones (CIFAR). Sede El Claustro. Bloque " $\mathrm{L}$ ", 4 piso Diag. 46A No. $15 \mathrm{~b}-10$ Editor, Arq. César Eligio-Triana

Teléfonos:

+57 (1) $3277300-3277333$

Ext. 3109; 3112 o 5146

Fax: +57 (1) 2858895
Correo electrónico: revistadearquitectura@ucatolica.edu.co cifar@ucatolica.edu.co

Página WEB: www.ucatolica.edu.co vínculo Revistas científicas

http://publicaciones.ucatolica.edu.co revistas-cientificas http://editorial.ucatolica.edu.co/ojsucatolica/revistas ucatolica/index.php/RevArq 
